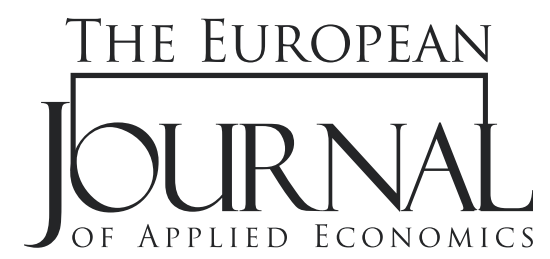

\title{
VOLATILITY SPILLOVER AND CONTAGION EFFECTS BETWEEN EURODOLLAR FUTURE AND ZERO COUPONS MARKETS: EVIDENCE FROM ITALY
}

\author{
Konstantinos Tsiaras* \\ University of loannina, \\ Greece
}

\begin{abstract}
:
This paper examines the time-varying conditional correlations between the Eurodollar futures market and the zero coupons of Banca Fideuram. We apply a bivariate dynamic conditional correlation (DCC) GARCH model in order to capture potential contagion effects between the markets for the period 2005-2017. Empirical results reveal contagion during the under-investigation period regarding the twenty-one bivariate models, showing that the Eurodollar futures market has a major impact on the zero coupons of Banca Fideuram. Findings have crucial implications for policymakers who provide regulations for the above-mentioned derivative markets.
\end{abstract}

\section{Article info:}

Received: Jun 3, 2020

Correction: July 31, 2020

Accepted: September 10, 2020

\section{Keywords:}

DCC-GARCH model, EURODOLLAR future market, zero coupons, financial contagion, dynamic conditional correlations.

\section{Jel Classification:}

C58, C61, G11, G15.

\section{INTRODUCTION}

This paper investigates the potential volatility spillover and contagion effects (Dimitriou, Kenourgios \& Simos 2013) of the Eurodollar futures market and the zero coupons of Banca Fideuram. We consider the zero coupons of Banca Fideuram ending from 2018 to 2033. By employing a bivariate DCC-GARCH model, we show significant volatility spillover effects (Sehgal, Ahmad \& Deisting 2015; Li \& Giles 2015; Aboura \& Chevallier 2015; Antonakakis, Floros \& Kizys 2016). Moreover, we use the definition of contagion as suggested by Forbes and Rigobon (2002). They defined contagion as a significant increase in cross-market linkages after a shock. Dynamic conditional correlations reveal contagion effects (Dimitriou \& Kenourgios 2015; Sensoy \& Hacihasanoglu 2015) in sub-periods between the Eurodollar futures market and all the zero coupons of Banca Fideuram. 
The motivation for this paper is analyzed as follows. Firstly, there is no other empirical research investigating the conditional second moments of the distribution between the Eurodollar futures market and the zero coupons of Banca Fideuram. Secondly, the potential existence of contagion between the Eurodollar futures market and the zero coupons of Banca Fideuram provides new evidence for financial theory. Thirdly, the under-investigation period is of great importance, since it entails major economic crises i.e., the financial crisis of 2008.

The paper is organized as follows. Section 2 presents the literature review and Section 3 provides the data characteristics. Section 4 provides the methodology. Section 5 shows the empirical results. The last section provides the conclusion.

\section{LITERATURE REVIEW}

There are numerous empirical studies investigating the spillovers among different future and financial markets (Mensi et al 2013; Kavussanos et al 2014; Li et al 2014; Antonakakis and Kizys 2015; Du and He 2015; Ewing and Malik 2016; Bagchi 2017; Roy and Roy 2017; Ma et al 2019; Tsiaras and Simos 2020; Tsiaras 2020, Tsiaras 2020).

Mensi et al (2013) find evidence of spillovers between the S\&P 500 and commodity price indices for energy, food, gold, and beverages over the turbulent period from 2000 to 2011.

Kavussanos et al (2014) examine the existence of spillover effects between commodity and freight markets for the period 2006-2009. By using different GARCH models, they show the existence of spillovers effects.

Li et al (2014) show potential spillovers and dynamic conditional correlations between spot and forward tanker freight markets. By using a multivariate GARCH model, they examine the period from 2006 to 2011.

Antonakakis and Kizys (2015) find evidence of volatility spillover effects between commodity and FOREX markets: crude oil, gold, silver, platinum, CHF/USD, GBP/USD, EUR.USD. They investigate the period 1987 to 2014 .

$\mathrm{Du}$ and He (2015) found evidence of significant spillover between crude oil and stock markets using daily data of the S\&P 500 stock index and West Texas Intermediate (WTI). Based on their results, they supported the existence of positive risk spillovers from stock to crude oil markets and negative spillovers from crude oil to stock markets.

Ewing and Malik (2016) examine the volatility of oil and US stock market prices incorporating structural breaks using daily data from 1996 to 2013. By employing univariate and bivariate GARCH models, they find no volatility spillovers between the two markets.

Bagchi (2017) investigates the dynamic relationship between crude oil price volatility and stock markets in the emerging economies like BRIC (Brazil, Russia, India and China) countries. By using a AR-APARCH model, he finds evidence of positive and negative relationships between the underinvestigation markets.

Roy and Roy (2017) show the financial contagion in Indian commodity derivative markets vis-à-vis bond, FOREX, gold, and stock markets. They applied a multivariate DCC-GARCH model for the period 2006-2016.

Ma et al (2019) examine the inter-connectedness between WTI oil price returns and the returns of listed firms in the US energy sector for the period 2008-2018. 
They show that, although idiosyncratic information is mostly independent of oil shocks, individual energy stock returns do respond to WTI price movements.

Tsiaras and Simos (2020) prove the spillover effects among S\&P 500, four national equity markets and the respective FOREX markets for the period from 2010 to 2018.

Tsiaras (2020) investigates and proves the spillovers between JPY/USD, KRW/USD, EUR/USD and INR/USD futures markets for the period 2014-2019. In our paper, we provide empirical evidence of spillover effects between major future FOREX market and Zero Coupons derivative markets.

To the best of our knowledge, there is no previous empirical evidence providing evidence of spillover effects between the under-investigation market.

\section{DATA CHARACTERISTICS}

We use daily data for Eurodollar futures market (DGCX-EUR/USD CONTINUOUS AVG.- SETT. PRICE) and sixteen zero coupons of Banca Fideuram (BANCA FIDEURAM ZERO CPN. 2018, BANCA FIDEURAM ZERO CPN. 2019, BANCA FIDEURAM ZERO CPN. 2020, BANCA FIDEURAM ZERO CPN. 2021, BANCA FIDEURAM ZERO CPN. 2022, BANCA FIDEURAM ZERO CPN. 2023, BANCA FIDEURAM ZERO CPN. 2024, BANCA FIDEURAM ZERO CPN. 2025, BANCA FIDEURAM ZERO CPN. 2026, BANCA FIDEURAM ZERO CPN. 2027, BANCA FIDEURAM ZERO CPN. 2028, BANCA FIDEURAM ZERO CPN. 2029, BANCA FIDEURAM ZERO CPN. 2030, BANCA FIDEURAM ZERO CPN. 2031, BANCA FIDEURAM ZERO CPN. 2032 and BANCA FIDEURAM ZERO CPN. 2033). We downloaded data from the Datastream database. We set the period from January 4, 2005 to December 11, 2017 (3375 observations). We use the market returns generated by the equation $r_{t}=\log \left(p_{t}\right)-\log \left(p_{t-1}\right)$, where $p_{t}$ is the price of future market on day $t$ and $p_{t-1}$ is the price of future market on day $t-1$.

In tables 1, 2, 3 and 4 we see the summary statistics for the markets returns. BANCA FIDEURAM ZERO CPN. 2032 exhibits the highest mean value (0,00023071). Based on the highest maximum $(0,077701)$, the second minimum $(-0,066133)$ and the second highest std. deviation $(0,0095707)$ values, BANCA FIDEURAM ZERO CPN. 2032 presents the largest fluctuations among all the markets. Additionally, all market returns are negatively skewed, except the cases of BANCA FIDEURAM ZERO CPN. 2018, BANCA FIDEURAM ZERO CPN. 2019, BANCA FIDEURAM ZERO CPN. 2020 and BANCA FIDEURAM ZERO CPN. 2021. Furthermore, we observe that all market returns show excess kurtosis. In addition, Jarque-Bera statistic results indicate the rejection of the null hypothesis of normality for all market returns. ADF (Dickey and Fuller 1979) test results reject the null hypotheses of unit root at $1 \%$ level, showing that the daily market returns appropriate for further testing. 
Table 1 - Summary Statistics of the Daily Market Logarithmic Returns

\begin{tabular}{|c|c|c|c|c|c|}
\hline & $\begin{array}{c}\text { DGCX- } \\
\text { EUR/USD } \\
\text { CONTINUOUS } \\
\text { AVG.- SETT. } \\
\text { PRICE }\end{array}$ & $\begin{array}{c}\text { BANCA } \\
\text { FIDEURAM } \\
\text { ZERO CPN. } \\
2018\end{array}$ & $\begin{array}{c}\text { BANCA } \\
\text { FIDEURAM } \\
\text { ZERO CPN. } \\
2019\end{array}$ & $\begin{array}{c}\text { BANCA } \\
\text { FIDEURAM } \\
\text { ZERO CPN. } \\
2020\end{array}$ & $\begin{array}{c}\text { BANCA } \\
\text { FIDEURAM } \\
\text { ZERO CPN. } \\
2021\end{array}$ \\
\hline Mean & $-3.62 \mathrm{e}-005$ & 0.00014653 & 0.00016334 & 0.00017082 & 0.00017633 \\
\hline Minimum & -0.034722 & -0.034328 & -0.037916 & -0.032092 & -0.036549 \\
\hline Maximum & 0.032842 & 0.052911 & 0.057069 & 0.057384 & 0.059144 \\
\hline Std. Deviation & 0.0058591 & 0.0040629 & 0.0042757 & 0.0050665 & 0.0054361 \\
\hline Skewness & -0.0067747 & $0.56365^{\star \star \star}$ & $0.59049^{\star * \star}$ & $0.66735^{\star * \star}$ & $0.23680^{\star * *}$ \\
\hline t-Statistic & 0.16075 & 13.374 & 14.011 & 15.835 & 5.6187 \\
\hline p-Value & 0.87229 & $8.5689 e-041$ & $1.3378 \mathrm{e}-044$ & $1.7961 \mathrm{e}-056$ & $1.9243 \mathrm{e}-008$ \\
\hline Excess Kyrtosis & $2.4752^{\star * \star *}$ & $16.889^{* * *}$ & $16.837^{\star \star *}$ & $13.692^{\star * *}$ & $9.9221^{\star * *}$ \\
\hline t-Statistic & 29.374 & 200.43 & 199.81 & 162.49 & 117.75 \\
\hline p-Value & $1.1804 \mathrm{e}-189$ & 0.00000 & 0.00000 & 0.00000 & 0.00000 \\
\hline Jarque-Bera & $861.58^{\star * *}$ & $40293^{\star * *}$ & $40062^{\star * *}$ & $26614^{* * *}$ & $13876^{\star * *}$ \\
\hline p-Value & $8.1273 e-188$ & 0.00000 & 0.00000 & 0.00000 & 0.00000 \\
\hline ADF Test & $-34.0035^{* * *}$ & $-36.1749^{* * *}$ & $-35.1774^{* * *}$ & $-35.273^{* * *}$ & $-35.4105^{\star * *}$ \\
\hline
\end{tabular}

Table 2 - Summary Statistics of the Daily Market Logarithmic Returns

\begin{tabular}{|c|c|c|c|c|}
\hline & $\begin{array}{c}\text { BANCA } \\
\text { FIDEURAM } \\
\text { ZERO CPN. } \\
2022\end{array}$ & $\begin{array}{c}\text { BANCA } \\
\text { FIDEURAM } \\
\text { ZERO CPN. } \\
2023\end{array}$ & $\begin{array}{c}\text { BANCA } \\
\text { FIDEURAM } \\
\text { ZERO CPN. } \\
2024\end{array}$ & $\begin{array}{c}\text { BANCA } \\
\text { FIDEURAM } \\
\text { ZERO CPN. } \\
2025\end{array}$ \\
\hline Mean & 0.0001646 & 0.00018798 & 0.0002024 & 0.00020046 \\
\hline Minimum & -0.036124 & -0.051395 & -0.048579 & -0.045075 \\
\hline Maximum & 0.059525 & 0.047033 & 0.049644 & 0.052734 \\
\hline Std. Deviation & 0.0058674 & 0.0062433 & 0.0066364 & 0.0066885 \\
\hline Skewness & $-0.14823^{\star * *}$ & $-0.10383^{\star \star \star}$ & $-0.23445^{\star * *}$ & $-0.17699^{* * *}$ \\
\hline t-Statistic & 3.5171 & 2.4636 & 5.5629 & 4.1996 \\
\hline p-Value & 0.00043625 & 0.013754 & $2.6528 \mathrm{e}-008$ & $2.6742 \mathrm{e}-005$ \\
\hline Excess Kyrtosis & $11.316^{* * *}$ & $8.4188^{* * *}$ & $6.8660^{* * *}$ & $6.0796^{* * *}$ \\
\hline t-Statistic & 134.29 & 99.909 & 81.481 & 72.149 \\
\hline p-Value & 0.00000 & 0.00000 & 0.00000 & 0.00000 \\
\hline Jarque-Bera & $18021^{\star * *}$ & $9973.1^{* * *}$ & $6660.3^{* * *}$ & $5215.4^{* * *}$ \\
\hline p-Value & 0.00000 & 0.00000 & 0.00000 & 0.00000 \\
\hline ADF Test & $-35.3086^{\star * *}$ & $-34.3199^{* * *}$ & $-35.7359^{* * *}$ & $-34.922^{* * *}$ \\
\hline
\end{tabular}


Table 3 - Summary Statistics of the Daily Market Logarithmic Returns

\begin{tabular}{|c|c|c|c|c|}
\hline & $\begin{array}{c}\text { BANCA } \\
\text { FIDEURAM } \\
\text { ZERO CPN. } \\
2026\end{array}$ & $\begin{array}{c}\text { BANCA } \\
\text { FIDEURAM } \\
\text { ZERO CPN. } \\
2027\end{array}$ & $\begin{array}{c}\text { BANCA } \\
\text { FIDEURAM } \\
\text { ZERO CPN. } \\
2028\end{array}$ & $\begin{array}{c}\text { BANCA } \\
\text { FIDEURAM } \\
\text { ZERO CPN. } \\
2029\end{array}$ \\
\hline Mean & 0.00020225 & 0.00020596 & 0.00020595 & 0.00021448 \\
\hline Minimum & -0.056538 & -0.057316 & -0.056162 & -0.06922 \\
\hline Maximum & 0.058081 & 0.046397 & 0.049962 & 0.051293 \\
\hline Std. Deviation & 0.0071898 & 0.0076207 & 0.0081711 & 0.0084838 \\
\hline Skewness & $-0.18968^{\star * *}$ & $-0.28312^{\star * *}$ & $-0.31747^{\star \star \star}$ & $-0.40512^{* * *}$ \\
\hline t-Statistic & 4.2871 & 6.7177 & 7.5328 & 9.6126 \\
\hline p-Value & $1.8099 \mathrm{e}-005$ & $1.8466 \mathrm{e}-011$ & $4.9673 \mathrm{e}-014$ & $7.0759 \mathrm{e}-022$ \\
\hline Excess Kyrtosis & $6.4695^{\star * *}$ & $5.4861^{\star * \star}$ & $5.1496^{* * *}$ & $5.6284^{\star * *}$ \\
\hline t-Statistic & 76.775 & 65.105 & 61.111 & 66.794 \\
\hline $\mathrm{p}$-Value & 0.00000 & 0.00000 & 0.00000 & 0.00000 \\
\hline Jarque-Bera & $5904.1^{* * *}$ & $4277.5^{\star * *}$ & $3785.8^{* * *}$ & $4547.1^{\star * *}$ \\
\hline p-Value & 0.00000 & 0.00000 & 0.00000 & 0.00000 \\
\hline ADF Test & $-34.5052^{\star * *}$ & $-35.2119^{* * *}$ & $-35.0801^{* * *}$ & $-35.9567^{\star * *}$ \\
\hline
\end{tabular}

Table 4 - Summary Statistics of the Daily Market Logarithmic Returns

\begin{tabular}{ccccc}
\hline & BANCA & BANCA & BANCA & BANCA \\
& $\begin{array}{c}\text { ZERO CPN. } \\
\mathbf{2 0 3 0}\end{array}$ & $\begin{array}{c}\text { ZERO CPN. } \\
\text { 2031 }\end{array}$ & $\begin{array}{c}\text { ZERO CPN. } \\
\mathbf{2 0 3 2}\end{array}$ & $\begin{array}{c}\text { ZERO CPN. } \\
\text { FIDEUR }\end{array}$ \\
\hline Mean & 0.00021384 & 0.00021572 & 0.00023071 & 0.00022541 \\
\hline Minimum & -0.062678 & -0.055032 & -0.066133 & -0.064688 \\
\hline Maximum & 0.059321 & 0.076138 & 0.077701 & 0.074629 \\
\hline Std. Deviation & 0.0087642 & 0.0092783 & 0.0095707 & 0.0097384 \\
\hline Skewness & $-0.20305^{* * *}$ & $-0.069956^{* *}$ & $-0.15964^{* * *}$ & $-0.14954^{* * *}$ \\
\hline t-Statistic & 4.8178 & 1.6599 & 3.7878 & 3.5482 \\
\hline p-Value & $1.4516 \mathrm{e}-006$ & 0.096938 & 0.00015197 & 0.00038786 \\
\hline Excess Kyrtosis & $5.6736^{* * *}$ & $5.0302^{* * *}$ & $5.8516^{* * *}$ & $5.4135^{* * *}$ \\
\hline t-Statistic & 67.330 & 59.694 & 69.443 & 64.244 \\
\hline p-Value & 0.00000 & 0.00000 & 0.00000 & 0.00000 \\
\hline Jarque-Bera & $4549.9^{* * *}$ & $3560.9^{* * *}$ & $4829.5^{* * *}$ & $4133.8^{* * *}$ \\
\hline p-Value & 0.00000 & 0.00000 & 0.00000 & 0.00000 \\
\hline ADF Test & $-34.9155^{* * *}$ & $-34.9721^{* * *}$ & $-35.1153^{* * *}$ & $-34.9907^{* * *}$ \\
\hline
\end{tabular}


Figure 1 graphs the logarithmic returns for DGCX-EUR/USD CONTINUOUS AVG.- SETT. PRICE, BANCA FIDEURAM ZERO CPN. 2018, BANCA FIDEURAM ZERO CPN. 2019, BANCA FIDEURAM ZERO CPN. 2020, BANCA FIDEURAM ZERO CPN. 2021, BANCA FIDEURAM ZERO CPN. 2022, BANCA FIDEURAM ZERO CPN. 2023, BANCA FIDEURAM ZERO CPN. 2024, BANCA FIDEURAM ZERO CPN. 2025, BANCA FIDEURAM ZERO CPN. 2026, BANCA FIDEURAM ZERO CPN. 2027, BANCA FIDEURAM ZERO CPN. 2028, BANCA FIDEURAM ZERO CPN. 2029, BANCA FIDEURAM ZERO CPN. 2030, BANCA FIDEURAM ZERO CPN. 2031, BANCA FIDEURAM ZERO CPN. 2032 and BANCA FIDEURAM ZERO CPN. 2033. Based on the virtual observation of the graph, we see time varying levels of fluctuations, indicating the presence of heteroskedasticity and appropriate the use of the DCC-GARCH model.

Figure 1 - Actual Series of the Logarithmic Returns of the Markets.

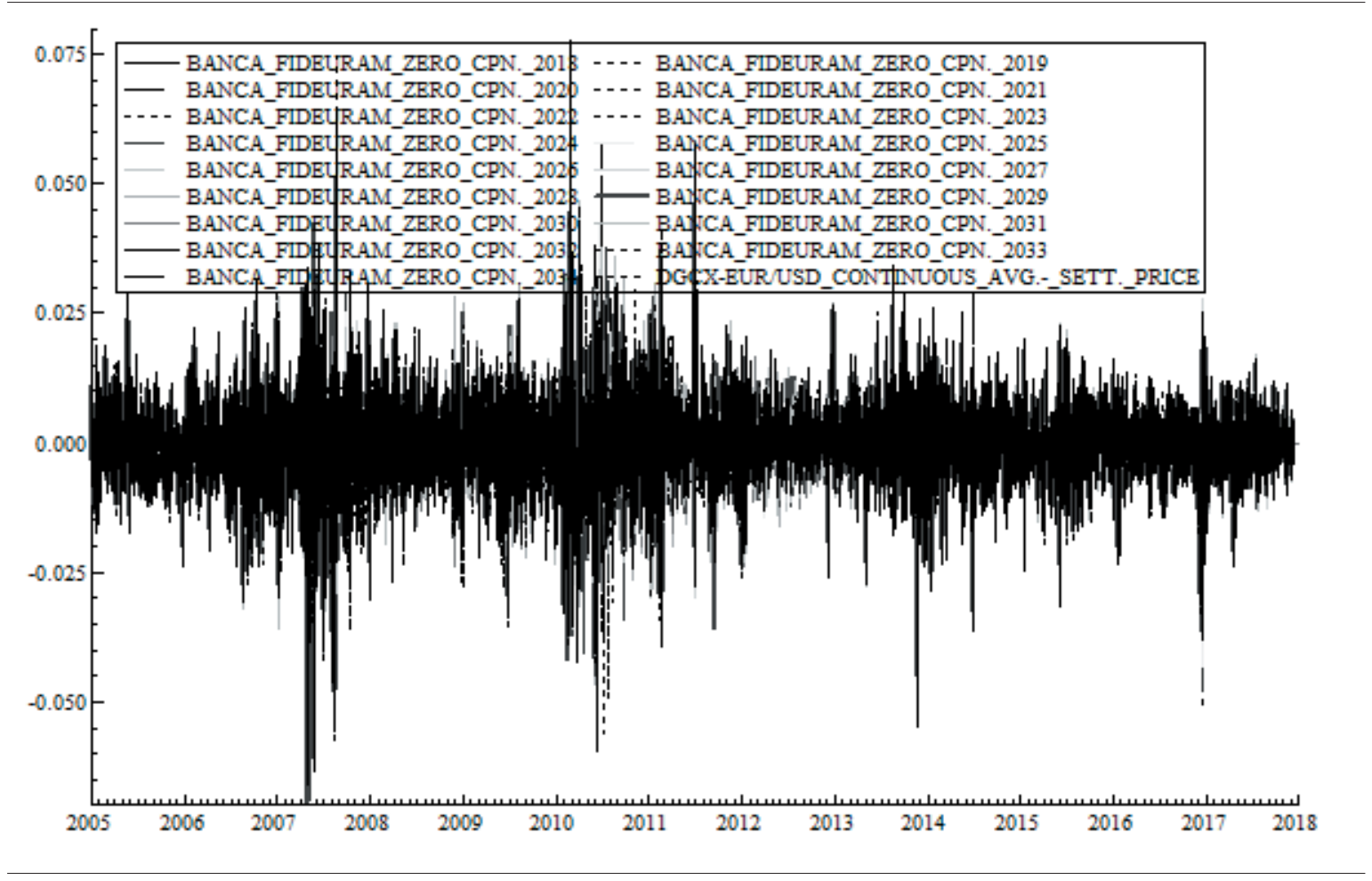

\section{METHODOLODY}

In the first stage, we generate the daily logarithmic returns:

$\mathrm{y}_{\mathrm{t}}=\mu+\varepsilon_{\mathrm{t}}$, with $t=1, \ldots, \mathrm{T}$

where $\mu$ is constant $\mathrm{y}_{\mathrm{t}}=\mu+\varepsilon_{\mathrm{t}}$ and $\boldsymbol{\varepsilon}_{\mathrm{t}}$ is standardized residuals, defined as follows:

$\varepsilon_{\mathrm{t}}=\sqrt{\mathrm{h}_{\mathrm{t}}} \mathrm{u}_{\mathrm{t}}$, where $\varepsilon_{\mathrm{t}} \sim \mathrm{N}\left(0, \mathrm{H}_{\mathrm{t}}\right)$ and $\mathrm{u}_{\mathrm{t}}$ are i.i.d.

where $\mathrm{u}_{\mathrm{t}}$ is standardized errors and $\mathrm{h}_{\mathrm{t}}$ is conditional variance depending on $\mathrm{h}_{\mathrm{t}}$ and $\boldsymbol{\varepsilon}_{\mathrm{t}}$ for each market lagged one period, generated by the univariate $\operatorname{GARCH}(1,1)$ model (Bollerslev 1986): 


$$
\mathrm{h}_{\mathrm{t}}=\omega+\mathrm{a} \varepsilon_{\mathrm{t}-1}^{2}+\mathrm{bh} \mathrm{t}-1_{1}
$$

where $\omega$ is constant, $a$ and $b$ are ARCH and GARCH effects.

In the second stage, we employ the Engle (2002) representation of the bivariate GARCH model inorder to estimate the bivariate conditional variance matrix $\left(H_{t}\right.$ is $\mathrm{N} \mathrm{x} \mathrm{N}$ matrix, with $\mathrm{N}$ the number of markets, $i=1, \ldots, \mathrm{N})$ as follows:

$$
\mathrm{H}_{\mathrm{t}}=\mathrm{D}_{\mathrm{t}} \mathrm{R}_{\mathrm{t}} \mathrm{D}_{\mathrm{t}}
$$

$\mathrm{D}_{\mathrm{t}}$ is the conditional variance matrix given by:

$$
\mathrm{D}_{\mathrm{t}}=\operatorname{diag}\left(\mathrm{h}_{11 \mathrm{t}}^{\frac{1}{2}} \ldots \mathrm{h}_{\mathrm{NN \textrm {t }}}^{\frac{1}{2}}\right)
$$

$\mathbf{R}_{t}$ is the condition correlation matrix of $\mathrm{N} \mathrm{x} \mathrm{N} \mathrm{dimension,} \mathrm{and} \mathrm{is} \mathrm{defined,} \mathrm{as} \mathrm{follows:}$

$$
\mathrm{R}_{\mathrm{t}}=\left(\rho_{\mathrm{iit}}\right)=\operatorname{diag}\left(\mathrm{q}_{11, \mathrm{t}}^{-\frac{1}{2}} \cdots \mathrm{q}_{\mathrm{NN}, \mathrm{t}}^{-\frac{1}{2}}\right) \mathrm{Q}_{\mathrm{t}} \operatorname{diag}\left(\mathrm{q}_{11, \mathrm{t}}^{-\frac{1}{2}} \cdots \mathrm{q}_{\mathrm{NN}, \mathrm{t}}^{-\frac{1}{2}}\right)
$$

where the $N$ x N symmetric positive definite matrix $Q_{t}=\left(q_{i i, t}\right)$ is given by:

$$
Q_{t}=(1-\alpha-\beta) \bar{Q}+\alpha u_{t-1} u_{t-1}^{\prime}+\beta Q_{t-1}
$$

$\overline{\mathrm{Q}}_{i}$ s the $\mathrm{N} x \mathrm{~N}$ unconditional variance matrix of $\mathrm{u}_{t}$, and $\alpha$ and $\beta$ are nonnegative scalar parameters, satisfying $\alpha+\beta<1$.

\section{EMPIRICAL RESULTS}

In this section, we present the empirical results generated by the multivariate DCC-GARCH model. Sub-section 5.1 shows the results of the univariate GARCH model, while in sub-section 5.2 we analyze the results of the multivariate DCC-GARCH model. In sub-section 5.3, we report an analysis of the generated Dynamic Conditional Correlations (DCCs).

\section{RESULTS OF THE UNIVARIATE GARCH $(1,1)$ MODEL}

Tables 5, 6, 7 and 8 show the estimated values for mean equation and univariate GARCH $(1,1)$ model. We observe statistically significant $\mu$ for all the market returns, except the case of DGCX-EUR/USD CONTINUOUS AVG.- SETT. PRICE. Additionally, empirical results report statistically significant $\omega$ for all the market returns. Moreover, ARCH (a) and GARCH (b) terms are highly significant for all the markets returns. 
Table 5 - Estimates of Univariate GARCH $(1,1)$ Model

\begin{tabular}{cccccc}
\hline & $\begin{array}{c}\text { DGCX- } \\
\text { EUR/USD } \\
\text { CONTINUOUS } \\
\text { AVG.- SETT. } \\
\text { PRICE }\end{array}$ & $\begin{array}{c}\text { FIDEURAM } \\
\text { ZERO CPN. } \\
\mathbf{2 0 1 8}\end{array}$ & $\begin{array}{c}\text { FIDEURAM } \\
\text { ZERO CPN. } \\
\mathbf{2 0 1 9}\end{array}$ & $\begin{array}{c}\text { FIDEURAM } \\
\text { ZERO CPN. } \\
\mathbf{2 0 2 0}\end{array}$ & $\begin{array}{c}\text { FIDEURAM } \\
\text { ZERO CPN. } \\
\text { 2021 }\end{array}$ \\
\hline constant $(\boldsymbol{\mu})$ & 0.0000377 & $0.0000221^{*}$ & $0.0000702^{* * *}$ & $0.0001333^{* * *}$ & $0.0001804^{* * *}$ \\
\hline t-Statistic & 0.4768 & 1.517 & 3.051 & 3.603 & 3.624 \\
\hline p-Value & 0.6335 & 0.1294 & 0.0023 & 0.0003 & 0.0003 \\
\hline constant $(\boldsymbol{\omega})$ & $0.050450^{*}$ & $0.001737^{*}$ & $0.005068^{*}$ & $0.016843^{*}$ & $0.040020^{*}$ \\
\hline t-Statistic & 1.107 & 1.288 & 1.370 & 1.464 & 1.478 \\
\hline p-Value & 0.2683 & 0.1979 & 0.1708 & 0.1433 & 0.1394 \\
\hline ARCH $(\boldsymbol{\alpha})$ & $0.037845^{* * *}$ & $0.114787^{* * *}$ & $0.099745^{* * *}$ & $0.081683^{* * *}$ & $0.070081^{* * *}$ \\
\hline t-Statistic & 7.898 & 4.879 & 3.732 & 3.699 & 3.345 \\
\hline p-Value & 0.0000 & 0.0000 & 0.0002 & 0.0002 & 0.0008 \\
\hline GARCH $(\boldsymbol{b})$ & $0.964238^{* * *}$ & $0.896551^{* * *}$ & $0.908039^{* * *}$ & $0.922360^{* * *}$ & $0.931229^{* * *}$ \\
\hline t-Statistic & 227.3 & 49.48 & 42.07 & 48.76 & 48.81 \\
\hline p-Value & 0.0000 & 0.0000 & 0.0000 & 0.0000 & 0.0000 \\
\hline
\end{tabular}

Table 6 - Estimates of Univariate GARCH $(1,1)$ Model

\begin{tabular}{ccccc}
\hline & BANCA & BANCA & BANCA & BANCA \\
& $\begin{array}{c}\text { FIDEURAM } \\
\text { ZER CPN. } \\
\text { 2022 }\end{array}$ & $\begin{array}{c}\text { FIDEURAM } \\
\text { ZERO CPN. } \\
\text { FIDEURAM }\end{array}$ & $\begin{array}{c}\text { ZERO CPN. } \\
\text { FIDEURAM }\end{array}$ & $\begin{array}{c}\text { ZERO CPN. } \\
\text { 2025 }\end{array}$ \\
\hline constant $(\boldsymbol{\mu})$ & $0.0002175^{* * *}$ & $0.0002566^{* * *}$ & $0.0002897^{* * *}$ & $0.0003358^{* * *}$ \\
\hline t-Statistic & 3.763 & 3.874 & 3.815 & 4.154 \\
\hline p-Value & 0.0002 & 0.0001 & 0.0001 & 0.0000 \\
\hline constant $(\boldsymbol{\omega})$ & $0.062064^{*}$ & $0.117230^{*}$ & $0.181788^{*}$ & $0.373052^{* *}$ \\
\hline t-Statistic & 1.628 & 1.878 & 1.656 & 1.990 \\
\hline p-Value & 0.1036 & 0.0604 & 0.0978 & 0.0466 \\
\hline ARCH $(\boldsymbol{\alpha})$ & $0.064147^{* * *}$ & $0.066864^{* * *}$ & $0.060533^{* * *}$ & $0.074506^{* * *}$ \\
\hline t-Statistic & 3.509 & 4.691 & 3.863 & 3.808 \\
\hline p-Value & 0.0005 & 0.0000 & 0.0001 & 0.0001 \\
\hline GARCH $(\boldsymbol{b})$ & $0.935513^{* * *}$ & $0.931312^{* * *}$ & $0.935657^{* * *}$ & $0.917570^{* * *}$ \\
\hline t-Statistic & 54.52 & 65.63 & 55.49 & 41.82 \\
\hline p-Value & 0.0000 & 0.00000 & 0.0000 & 0.0000 \\
\hline
\end{tabular}


Table 7 - Estimates of Univariate GARCH $(1,1)$ Model

\begin{tabular}{|c|c|c|c|c|}
\hline & $\begin{array}{c}\text { BANCA } \\
\text { FIDEURAM } \\
\text { ZERO CPN. } \\
2026\end{array}$ & $\begin{array}{c}\text { BANCA } \\
\text { FIDEURAM } \\
\text { ZERO CPN. } \\
2027\end{array}$ & $\begin{array}{c}\text { BANCA } \\
\text { FIDEURAM } \\
\text { ZERO CPN. } \\
2028\end{array}$ & $\begin{array}{c}\text { BANCA } \\
\text { FIDEURAM } \\
\text { ZERO CPN. } \\
2029\end{array}$ \\
\hline constant $(\mu)$ & $0.0003728^{\star \star \star}$ & $0.0003899^{* * *}$ & $0.0003379^{* * *}$ & $0.000410^{\star * *}$ \\
\hline t-Statistic & 4.199 & 4.145 & 3.702 & 3.874 \\
\hline p-Value & 0.0000 & 0,0000 & 0.0002 & 0.0001 \\
\hline constant $(\omega)$ & $0.585090^{\star * *}$ & $0.865811^{\star * *}$ & $0.803986^{\star * *}$ & $1.664445^{\star * *}$ \\
\hline t-Statistic & 2.282 & 2.165 & 2.104 & 2.049 \\
\hline p-Value & 0.0225 & 0.0304 & 0.0354 & 0.0406 \\
\hline $\operatorname{ARCH}(\alpha)$ & $0.078349^{* * *}$ & $0.086326^{\star * *}$ & $0.075854^{\star \star \star}$ & $0.101611^{\star \star \star}$ \\
\hline t-Statistic & 4.625 & 4.194 & 4.193 & 3.700 \\
\hline p-Value & 0.0000 & 0.0000 & 0.0000 & 0.0002 \\
\hline GARCH (b) & $0.910445^{\star * \star}$ & $0.898462^{\star * *}$ & $0.911873^{\star \star *}$ & $0.874166^{\star * *}$ \\
\hline t-Statistic & 44.88 & 34.42 & 40.40 & 23.27 \\
\hline p-Value & 0.0000 & 0.0000 & 0.0000 & 0.0000 \\
\hline
\end{tabular}

Table 8 - Estimates of Univariate GARCH $(1,1)$ Model

\begin{tabular}{|c|c|c|c|c|}
\hline & $\begin{array}{c}\text { BANCA } \\
\text { FIDEURAM } \\
\text { ZERO CPN. } \\
2030\end{array}$ & $\begin{array}{c}\text { BANCA } \\
\text { FIDEURAM } \\
\text { ZERO CPN. } \\
2031\end{array}$ & $\begin{array}{c}\text { BANCA } \\
\text { FIDEURAM } \\
\text { ZERO CPN. } \\
2032\end{array}$ & $\begin{array}{c}\text { BANCA } \\
\text { FIDEURAM } \\
\text { ZERO CPN. } \\
2033\end{array}$ \\
\hline constant $(\mu)$ & $0,000423^{\star * *}$ & $0.000457^{\star * *}$ & $0.000462^{\star * *}$ & $0.000448^{\star * *}$ \\
\hline t-Statistic & 3.785 & 3.884 & 3.786 & 3.596 \\
\hline p-Value & 0.0002 & 0.0001 & 0.0002 & 0.0003 \\
\hline constant $(\omega)$ & $1.354293^{\star *}$ & $1.555353^{* * *}$ & $1.497339^{* * *}$ & $1.648639^{* * *}$ \\
\hline t-Statistic & 1.903 & 2.129 & 2.453 & 2.399 \\
\hline p-Value & 0.0571 & 0.0334 & 0.0142 & 0.0165 \\
\hline $\operatorname{ARCH}(\alpha)$ & $0.083461^{\star \star *}$ & $0.088127^{\star * *}$ & $0.080689^{\star * *}$ & $0.082399^{\star * *}$ \\
\hline t-Statistic & 3.603 & 4.198 & 4.700 & 4.553 \\
\hline p-Value & 0.0003 & 0.0000 & 0.0000 & 0.0000 \\
\hline GARCH (b) & $0.898062^{\star * *}$ & $0.893645^{\star * *}$ & $0.902314^{* * *}$ & $0.899750^{* * *}$ \\
\hline t-Statistic & 28.59 & 31.76 & 40.47 & 37.88 \\
\hline p-Value & 0.0000 & 0.0000 & 0.0000 & 0.0000 \\
\hline
\end{tabular}


In figure 2, we observe the behavior of conditional variances for DGCX-EUR/USD CONTINUOUS AVG.- SETT. PRICE, BANCA FIDEURAM ZERO CPN. 2018, BANCA FIDEURAM ZERO CPN. 2019, BANCA FIDEURAM ZERO CPN. 2020, BANCA FIDEURAM ZERO CPN. 2021, BANCA FIDEURAM ZERO CPN. 2022, BANCA FIDEURAM ZERO CPN. 2023, BANCA FIDEURAM ZERO CPN. 2024, BANCA FIDEURAM ZERO CPN. 2025, BANCA FIDEURAM ZERO CPN. 2026, BANCA FIDEURAM ZERO CPN. 2027, BANCA FIDEURAM ZERO CPN. 2028, BANCA FIDEURAM ZERO CPN. 2029, BANCA FIDEURAM ZERO CPN. 2030, BANCA FIDEURAM ZERO CPN. 2031, BANCA FIDEURAM ZERO CPN. 2032, and BANCA FIDEURAM ZERO CPN. 2033. We see strongly volatile conditional variances for all the market returns over time. Additionally, results indicate a common movement of conditional volatilities.

Figure 2 - Conditional Variances of the Univariate GARCH $(1,1)$ Model.

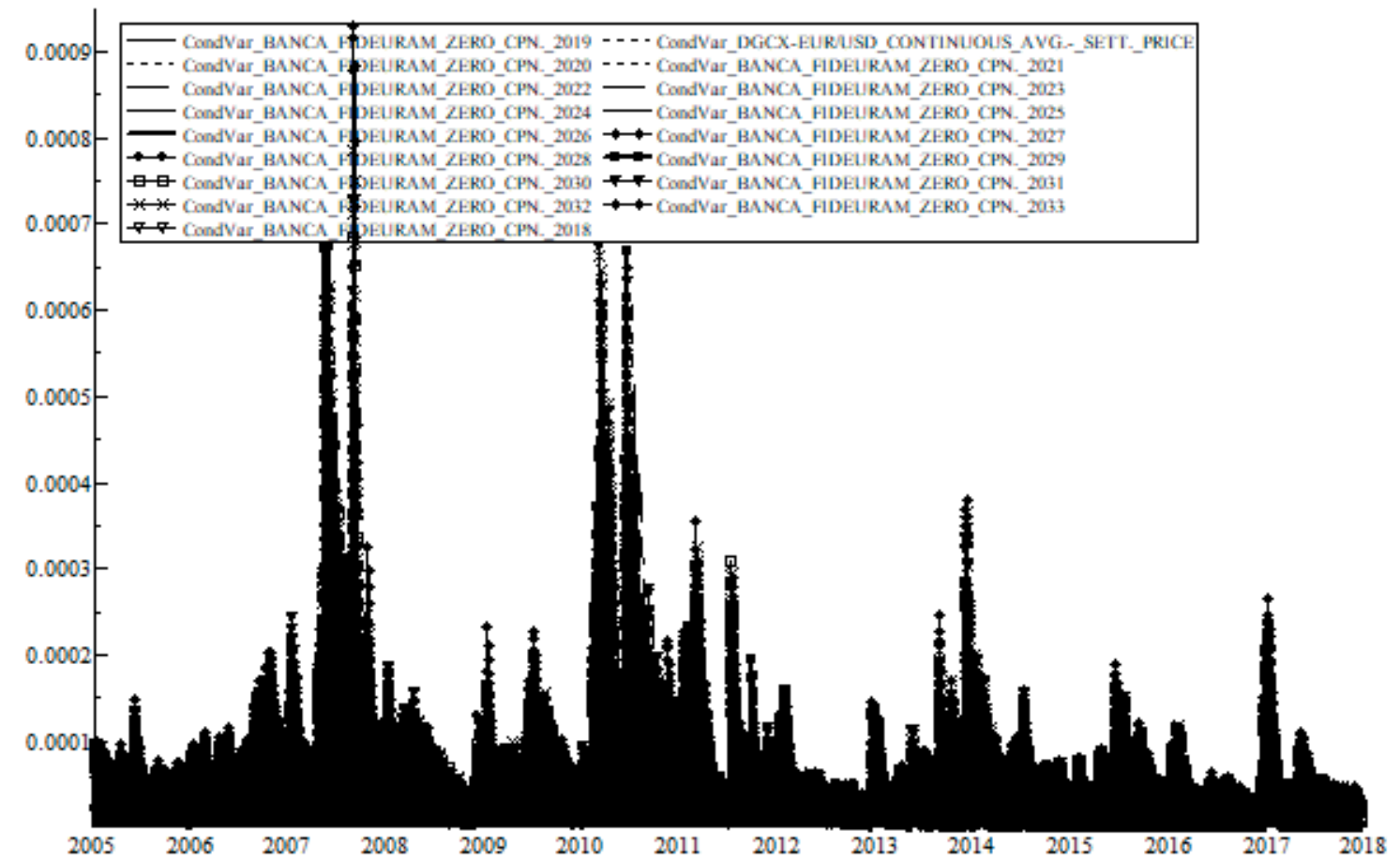

\section{RESULTS OF THE BIVARIATE DCC-GARCH $(1,1)$ MODEL, DIAGNOSTIC TESTS AND SELECTED INFORMATION CRITERIA}

Tables 9, 10, 11 and 12 present the results of the bivariate DCC model estimations. We observe that the average CORij is statistically significant for the pairs of markets: DGCX-EUR/USD CONTINUOUS AVG.- SETT. PRICE - BANCA FIDEURAM ZERO CPN. 2027, DGCX-EUR/USD CONTINUOUS AVG.- SETT. PRICE - BANCA FIDEURAM ZERO CPN. 2029 and DGCX-EUR/USD CONTINUOUS AVG.- SETT. PRICE - BANCA FIDEURAM ZERO CPN. 2032. Furthermore, we see statistically significant $\boldsymbol{\alpha}$ and $\boldsymbol{\beta}$ parameters, indicating strong ARCH and GARCH effects for all the pairs of market returns (Efimova and Serletis 2014; Li and Giles 2014; Sehgal and Ghosh 2016; Chang et al 2018; Sun et al 2019; Sukhonpitumart et al 2020; Yu et al 2020; Belhassine 2020). Additionally, we provide the estimates of the degrees of freedom (v) and of the log-likelihood. 
Table 9 - Estimates of the Bivariate DCC-GARCH (1,1) Model, Degrees of Freedom, Log-likelihood

\begin{tabular}{|c|c|c|c|c|}
\hline & $\begin{array}{c}\text { DGCX- } \\
\text { EUR/USD } \\
\text { CONTINUOUS } \\
\text { AVG.- SETT. } \\
\text { PRICE - BANCA } \\
\text { FIDEURAM } \\
\text { ZERO CPN. } \\
2018\end{array}$ & $\begin{array}{c}\text { DGCX- } \\
\text { EUR/USD } \\
\text { CONTINUOUS } \\
\text { AVG.- SETT. } \\
\text { PRICE - BANCA } \\
\text { FIDEURAM } \\
\text { ZERO CPN. } \\
2019\end{array}$ & $\begin{array}{c}\text { DGCX- } \\
\text { EUR/USD } \\
\text { CONTINUOUS } \\
\text { AVG.- SETT. } \\
\text { PRICE - BANCA } \\
\text { FIDEURAM } \\
\text { ZERO CPN. } \\
2020\end{array}$ & $\begin{array}{c}\text { DGCX- } \\
\text { EUR/USD } \\
\text { CONTINUOUS } \\
\text { AVG.- SETT. } \\
\text { PRICE - BANCA } \\
\text { FIDEURAM } \\
\text { ZERO CPN. } \\
2021\end{array}$ \\
\hline Average CORij & -0.028369 & -0.029169 & -0.26245 & -0.028106 \\
\hline t-Statistic & -0.7921 & -0.8427 & -0.7534 & -0.7739 \\
\hline p-Value & 0.4284 & 0.3995 & 0.4513 & 0.4390 \\
\hline alpha $(\alpha)$ & $0.008466^{\star * *}$ & $0.010216^{\star * *}$ & $0.008200^{\star * *}$ & $0.008050^{\star * *}$ \\
\hline t-Statistic & 3.041 & 3.571 & 3.473 & 3.449 \\
\hline p-Value & 0.0024 & 0.0004 & 0.0005 & 0.0006 \\
\hline beta $(\beta)$ & $0.983542^{\star \star \star *}$ & $0.979266^{\star * \star}$ & $0.983939^{\star * *}$ & $0.984555^{\text {} \star * *}$ \\
\hline t-Statistic & 179.9 & 185.2 & 226.1 & 240.0 \\
\hline p-Value & 0.0000 & 0.0000 & 0.0000 & 0.0000 \\
\hline $\begin{array}{l}\text { degrees of } \\
\text { freedom }(d f)\end{array}$ & $5.730267^{\star * \star}$ & $5.875022^{\star * *}$ & $6.500954^{\star * *}$ & $6.450702^{\star * \star}$ \\
\hline t-Statistic & 12.31 & 12.45 & 12.39 & 12.46 \\
\hline p-Value & 0.0000 & 0.0000 & 0.0000 & 0.0000 \\
\hline log-likelihood & 28728.993 & 28046.932 & 27161.298 & 26627.008 \\
\hline
\end{tabular}

Table 10 - Estimates of the Bivariate DCC-GARCH (1,1) Model, Degrees of Freedom, Log-likelihood

\begin{tabular}{|c|c|c|c|c|}
\hline & $\begin{array}{c}\text { DGCX- } \\
\text { EUR/USD } \\
\text { CONTINUOUS } \\
\text { AVG.- SETT. } \\
\text { PRICE - BANCA } \\
\text { FIDEURAM } \\
\text { ZERO CPN. } \\
2022\end{array}$ & $\begin{array}{c}\text { DGCX- } \\
\text { EUR/USD } \\
\text { CONTINUOUS } \\
\text { AVG.- SETT. } \\
\text { PRICE - BANCA } \\
\text { FIDEURAM } \\
\text { ZERO CPN. } \\
2023\end{array}$ & $\begin{array}{c}\text { DGCX- } \\
\text { EUR/USD } \\
\text { CONTINUOUS } \\
\text { AVG.- SETT. } \\
\text { PRICE - BANCA } \\
\text { FIDEURAM } \\
\text { ZERO CPN. } \\
2024\end{array}$ & $\begin{array}{c}\text { DGCX- } \\
\text { EUR/USD } \\
\text { CONTINUOUS } \\
\text { AVG.- SETT. } \\
\text { PRICE - BANCA } \\
\text { FIDEURAM } \\
\text { ZERO CPN. } \\
2025\end{array}$ \\
\hline Average CORij & -0.032175 & -0.029485 & -0.026158 & -0.030716 \\
\hline t-Statistic & -0.9473 & -0.8569 & -0.7185 & -0.8789 \\
\hline p-Value & 0.3435 & 0.3915 & 0.4725 & 0.3795 \\
\hline alpha $(\alpha)$ & $0.007305^{\star \star *}$ & $0.008021^{\star \star \star}$ & $0.008913^{\star \star *}$ & $0.007824^{\star * \star}$ \\
\hline t-Statistic & 3.280 & 3.461 & 3.563 & 3.340 \\
\hline p-Value & 0.0010 & 0.0005 & 0.0004 & 0.0008 \\
\hline beta $(\beta)$ & $0.984699^{\star * *}$ & $0.983735^{\star \star *}$ & $0.982894^{\star * *}$ & $0.984395^{\star \star \star}$ \\
\hline t-Statistic & 233.1 & 245.0 & 215.6 & 216.9 \\
\hline p-Value & 0.0000 & 0.0000 & 0.0000 & 0.0000 \\
\hline $\begin{array}{l}\text { degrees of } \\
\text { freedom }(d f)\end{array}$ & $6.448894^{* * *}$ & $6.700370^{\star * *}$ & $6.856377^{* * *}$ & $7.074205^{\star * \star}$ \\
\hline t-Statistic & 12.41 & 11.73 & 11.79 & 12.19 \\
\hline p-Value & 0.0000 & 0.0000 & 0.0000 & 0.0000 \\
\hline log-likelihood & 26326.015 & 26048.641 & 25727.520 & 25603.802 \\
\hline
\end{tabular}


Table 11 - Estimates of the Bivariate DCC-GARCH (1,1) Model, Degrees of Freedom, Log-likelihood

\begin{tabular}{|c|c|c|c|c|}
\hline & $\begin{array}{c}\text { DGCX- } \\
\text { EUR/USD } \\
\text { CONTINUOUS } \\
\text { AVG.- SETT. } \\
\text { PRICE - BANCA } \\
\text { FIDEURAM } \\
\text { ZERO CPN. } \\
2026\end{array}$ & $\begin{array}{c}\text { DGCX- } \\
\text { EUR/USD } \\
\text { CONTINUOUS } \\
\text { AVG.- SETT. } \\
\text { PRICE - BANCA } \\
\text { FIDEURAM } \\
\text { ZERO CPN. } \\
2027\end{array}$ & $\begin{array}{c}\text { DGCX- } \\
\text { EUR/USD } \\
\text { CONTINUOUS } \\
\text { AVG.- SETT. } \\
\text { PRICE - BANCA } \\
\text { FIDEURAM } \\
\text { ZERO CPN. } \\
2028\end{array}$ & $\begin{array}{c}\text { DGCX- } \\
\text { EUR/USD } \\
\text { CONTINUOUS } \\
\text { AVG.- SETT. } \\
\text { PRICE - BANCA } \\
\text { FIDEURAM } \\
\text { ZERO CPN. } \\
2029\end{array}$ \\
\hline Average CORij & -0.021714 & $-0.036694^{*}$ & -0.020921 & $-0.034145^{*}$ \\
\hline t-Statistic & -0.6194 & -1.080 & -0.6453 & -1.064 \\
\hline p-Value & 0.5357 & 0.2803 & 0.5188 & 0.2872 \\
\hline alpha $(\alpha)$ & $0.007685^{\star * *}$ & $0.007194^{\star * *}$ & $0.006878^{\star * *}$ & $0.007204^{\star * *}$ \\
\hline t-Statistic & 3.413 & 3.301 & 3.168 & 3.132 \\
\hline p-Value & 0.0006 & 0.0010 & 0.0015 & 0.0018 \\
\hline beta $(\beta)$ & $0.984614^{* * *}$ & $0.985149^{* * *}$ & $0.985076^{* * *}$ & $0.984160^{* * *}$ \\
\hline t-Statistic & 243.9 & 253.2 & 243.3 & 213.3 \\
\hline p-Value & 0.0000 & 0.0000 & 0.0000 & 0.0000 \\
\hline $\begin{array}{c}\text { degrees of } \\
\text { freedom }(d f)\end{array}$ & $6.853648^{* * *}$ & $6.925275^{\star * *}$ & $7.264400^{* * *}$ & $7.044709^{* * *}$ \\
\hline t-Statistic & 12.27 & 11.90 & 11.70 & 11.62 \\
\hline p-Value & 0.0000 & 0.0000 & 0.0000 & 0.0000 \\
\hline log-likelihood & 25334.343 & 25132.457 & 24870.512 & 24754.147 \\
\hline
\end{tabular}

Table 12 - Estimates of the Bivariate DCC-GARCH (1,1) Model, Degrees of Freedom, Log-likelihood

\begin{tabular}{|c|c|c|c|c|}
\hline & $\begin{array}{c}\text { DGCX- } \\
\text { EUR/USD } \\
\text { CONTINUOUS } \\
\text { AVG.- SETT. } \\
\text { PRICE - BANCA } \\
\text { FIDEURAM } \\
\text { ZERO CPN. } \\
2026\end{array}$ & $\begin{array}{c}\text { DGCX- } \\
\text { EUR/USD } \\
\text { CONTINUOUS } \\
\text { AVG.- SETT. } \\
\text { PRICE - BANCA } \\
\text { FIDEURAM } \\
\text { ZERO CPN. } \\
2027\end{array}$ & $\begin{array}{c}\text { DGCX- } \\
\text { EUR/USD } \\
\text { CONTINUOUS } \\
\text { AVG.- SETT. } \\
\text { PRICE - BANCA } \\
\text { FIDEURAM } \\
\text { ZERO CPN. } \\
2028\end{array}$ & $\begin{array}{c}\text { DGCX- } \\
\text { EUR/USD } \\
\text { CONTINUOUS } \\
\text { AVG.- SETT. } \\
\text { PRICE - BANCA } \\
\text { FIDEURAM } \\
\text { ZERO CPN. } \\
2029\end{array}$ \\
\hline Average CORij & -0.030906 & -0.029990 & $-0.036048^{*}$ & -0.029070 \\
\hline t-Statistic & -0.9881 & -0.9409 & -1.147 & -0.8904 \\
\hline p-Value & 0.3232 & 0.3468 & 0.2513 & 0.3733 \\
\hline alpha $(\alpha)$ & $0.007099^{\star * *}$ & $0.007192^{* * *}$ & $0.006640^{\star * *}$ & $0.007193^{\star * *}$ \\
\hline t-Statistic & 3.039 & 2.999 & 3.031 & 3.168 \\
\hline p-Value & 0.0024 & 0.0027 & 0.0025 & 0.0015 \\
\hline beta $(\beta)$ & $0.983747^{\star * *}$ & $0.984130^{* * *}$ & $0.984981^{\star * *}$ & $0.984612^{* * *}$ \\
\hline t-Statistic & 209.6 & 196.7 & 222.6 & 214.6 \\
\hline p-Value & 0.0000 & 0.0000 & 0.0000 & 0.0000 \\
\hline $\begin{array}{c}\text { degrees of } \\
\text { freedom }(d f)\end{array}$ & $7.029137^{\star \star * *}$ & $7.042781^{\star * *}$ & $6.986029^{* * *}$ & $7.010423^{* * *}$ \\
\hline t-Statistic & 11.65 & 11.62 & 11.84 & 12.00 \\
\hline p-Value & 0.0000 & 0.0000 & 0.0000 & 0.0000 \\
\hline log-likelihood & 24602.282 & 24394.485 & 24298.304 & 24222.677 \\
\hline
\end{tabular}


In tables $13,14,15$, and 16 , we report the results of the diagnostic tests and information criteria. $\boldsymbol{x}^{2}(4)$ statistic results suggest that the null hypothesis of no spillovers is rejected at $1 \%$ significance level. Ljuing-Box test results (Hosking, 1980; Li-McLeod, 1983) provide evidence of no serial autocorrelation, suggesting the absence of misspecification errors of the estimated multivariate GARCH model. Moreover, the estimated AIC and SIC information criteria are presented.

Table 13 - Diagnostic Tests and Information Criteria

\begin{tabular}{|c|c|c|c|c|}
\hline & $\begin{array}{c}\text { DGCX- } \\
\text { EUR/USD } \\
\text { CONTINUOUS } \\
\text { AVG.- SETT. } \\
\text { PRICE - BANCA } \\
\text { FIDEURAM } \\
\text { ZERO CPN. } \\
2018\end{array}$ & $\begin{array}{c}\text { DGCX- } \\
\text { EUR/USD } \\
\text { CONTINUOUS } \\
\text { AVG.- SETT. } \\
\text { PRICE - BANCA } \\
\text { FIDEURAM } \\
\text { ZERO CPN. } \\
2019\end{array}$ & $\begin{array}{c}\text { DGCX- } \\
\text { EUR/USD } \\
\text { CONTINUOUS } \\
\text { AVG.- SETT. } \\
\text { PRICE - BANCA } \\
\text { FIDEURAM } \\
\text { ZERO CPN. } \\
2020\end{array}$ & $\begin{array}{c}\text { DGCX- } \\
\text { EUR/USD } \\
\text { CONTINUOUS } \\
\text { AVG.- SETT. } \\
\text { PRICE - BANCA } \\
\text { FIDEURAM } \\
\text { ZERO CPN. } \\
2021 \\
\end{array}$ \\
\hline$x^{2}(4)$ & $8610.8^{\star *}$ & $7362.6^{* *}$ & $1550.4^{\star *}$ & $1156.0^{* *}$ \\
\hline p-Value & 0.0000 & 0.0000 & 0.0000 & 0.0000 \\
\hline Hosking (50) & 202.361 & 221.370 & 211.544 & 219.100 \\
\hline p-Value & 0.4400240 & 0.1432761 & 0.2743670 & 0.1687156 \\
\hline Hosking $^{2}$ (50) & 171.515 & 166.338 & 226.453 & 230.384 \\
\hline p-Value & 0.9133156 & 0.9506138 & 0.0808038 & 0.0571307 \\
\hline Li-McLeod (50) & 202.575 & 221.452 & 211.587 & 219.146 \\
\hline p-Value & 0.4358555 & 0.1424173 & 0.2736908 & 0.1681628 \\
\hline Li-McLeod $^{2}(50)$ & 172.026 & 166.843 & 226.539 & 230.334 \\
\hline p-Value & 0.9087771 & 0.9476324 & 0.0802170 & 0.0573922 \\
\hline Akaike & 0.002067 & 0.002187 & 0.002342 & 0.002436 \\
\hline Schwarz & 0.023842 & 0.023961 & 0.024117 & 0.024211 \\
\hline
\end{tabular}

Table 14 - Diagnostic Tests and Information Criteria

\begin{tabular}{|c|c|c|c|c|}
\hline & $\begin{array}{c}\text { DGCX- } \\
\text { EUR/USD } \\
\text { CONTINUOUS } \\
\text { AVG.- SETT. } \\
\text { PRICE - BANCA } \\
\text { FIDEURAM } \\
\text { ZERO CPN. } \\
2022\end{array}$ & $\begin{array}{c}\text { DGCX- } \\
\text { EUR/USD } \\
\text { CONTINUOUS } \\
\text { AVG.- SETT. } \\
\text { PRICE - BANCA } \\
\text { FIDEURAM } \\
\text { ZERO CPN. } \\
2023\end{array}$ & $\begin{array}{c}\text { DGCX- } \\
\text { EUR/USD } \\
\text { CONTINUOUS } \\
\text { AVG.- SETT. } \\
\text { PRICE - BANCA } \\
\text { FIDEURAM } \\
\text { ZERO CPN. } \\
2024\end{array}$ & $\begin{array}{c}\text { DGCX- } \\
\text { EUR/USD } \\
\text { CONTINUOUS } \\
\text { AVG.- SETT. } \\
\text { PRICE - BANCA } \\
\text { FIDEURAM } \\
\text { ZERO CPN. } \\
2025\end{array}$ \\
\hline$x^{2}(4)$ & $1970.3^{* *}$ & $2510,7^{\star \star}$ & $1463.2^{* *}$ & $688.23^{\star *}$ \\
\hline p-Value & 0.0000 & 0.0000 & 0.0000 & 0.0000 \\
\hline Hosking (50) & 221.416 & 202.490 & 204.207 & 195.549 \\
\hline p-Value & 0.1427906 & 0.4375132 & 0.4043224 & 0.5756642 \\
\hline Hosking $^{2}$ (50) & 201.280 & 181.741 & 194.714 & 191.925 \\
\hline p-Value & 0.4217686 & 0.7901022 & 0.5527005 & 0.6082992 \\
\hline Li-McLeod (50) & 221.507 & 202.618 & 204.279 & 195.733 \\
\hline p-Value & 0.1418376 & 0.4350033 & 0.4029490 & 0.5720145 \\
\hline Li-McLeod $^{2}$ (50) & 201.571 & 182.022 & 194.924 & 192.107 \\
\hline p-Value & 0.4161116 & 0.7857841 & 0.5484897 & 0.6046953 \\
\hline Akaike & 0.002489 & 0.002537 & 0.002594 & 0.002616 \\
\hline Schwarz & 0.024263 & 0.024312 & 0.024369 & 0.024390 \\
\hline
\end{tabular}


Table 15 - Diagnostic Tests and Information Criteria

\begin{tabular}{|c|c|c|c|c|}
\hline & $\begin{array}{c}\text { DGCX- } \\
\text { EUR/USD } \\
\text { CONTINUOUS } \\
\text { AVG.- SETT. } \\
\text { PRICE - BANCA } \\
\text { FIDEURAM } \\
\text { ZERO CPN. } \\
2026\end{array}$ & $\begin{array}{c}\text { DGCX- } \\
\text { EUR/USD } \\
\text { CONTINUOUS } \\
\text { AVG.- SETT. } \\
\text { PRICE - BANCA } \\
\text { FIDEURAM } \\
\text { ZERO CPN. } \\
2027\end{array}$ & $\begin{array}{c}\text { DGCX- } \\
\text { EUR/USD } \\
\text { CONTINUOUS } \\
\text { AVG.- SETT. } \\
\text { PRICE - BANCA } \\
\text { FIDEURAM } \\
\text { ZERO CPN. } \\
2028\end{array}$ & $\begin{array}{c}\text { DGCX- } \\
\text { EUR/USD } \\
\text { CONTINUOUS } \\
\text { AVG.- SETT. } \\
\text { PRICE - BANCA } \\
\text { FIDEURAM } \\
\text { ZERO CPN. } \\
2029\end{array}$ \\
\hline$x^{2}(4)$ & $850.54^{\star *}$ & $1487.7^{\star *}$ & $688.45^{\star \star}$ & $11490144.8^{* *}$ \\
\hline p-Value & 0.0000 & 0.0000 & 0.0000 & 0.0000 \\
\hline Hosking (50) & 189.425 & 189.649 & 198.376 & 191.851 \\
\hline p-Value & 0.6931695 & 0.6890983 & 0.5191595 & 0.6479014 \\
\hline Hosking ${ }^{2}(50)$ & 210.468 & 205.508 & 211.294 & 218.450 \\
\hline p-Value & 0.2587621 & 0.3423628 & 0.2460402 & 0.1520976 \\
\hline Li-McLeod (50) & 189.609 & 189.939 & 198.546 & 192.042 \\
\hline p-Value & 0.6898251 & 0.6856063 & 0.5157553 & 0.6442619 \\
\hline Li-McLeod $^{2}$ (50) & 210.356 & 205.489 & 211.277 & 218.314 \\
\hline p-Value & 0.2605135 & 0.3427115 & 0.2463003 & 0.1536092 \\
\hline Akaike & 0.002663 & 0.002698 & 0.002744 & 0.002765 \\
\hline Schwarz & 0.024438 & 0.024473 & 0.024519 & 0.024539 \\
\hline
\end{tabular}

Table 16 - Diagnostic Tests and Information Criteria

\begin{tabular}{|c|c|c|c|c|}
\hline & $\begin{array}{c}\text { DGCX- } \\
\text { EUR/USD } \\
\text { CONTINUOUS } \\
\text { AVG.- SETT. } \\
\text { PRICE - BANCA } \\
\text { FIDEURAM } \\
\text { ZERO CPN. } \\
2030\end{array}$ & $\begin{array}{c}\text { DGCX- } \\
\text { EUR/USD } \\
\text { CONTINUOUS } \\
\text { AVG.- SETT. } \\
\text { PRICE - BANCA } \\
\text { FIDEURAM } \\
\text { ZERO CPN. } \\
2031\end{array}$ & $\begin{array}{c}\text { DGCX- } \\
\text { EUR/USD } \\
\text { CONTINUOUS } \\
\text { AVG.- SETT. } \\
\text { PRICE - BANCA } \\
\text { FIDEURAM } \\
\text { ZERO CPN. } \\
2032\end{array}$ & $\begin{array}{c}\text { DGCX- } \\
\text { EUR/USD } \\
\text { CONTINUOUS } \\
\text { AVG.- SETT. } \\
\text { PRICE - BANCA } \\
\text { FIDEURAM } \\
\text { ZERO CPN. } \\
2033\end{array}$ \\
\hline$x^{2}(4)$ & $1615.6^{\star *}$ & $1320.5^{\star \star}$ & $1265,8^{\star *}$ & $898,61^{\star *}$ \\
\hline p-Value & 0.0000 & 0.0000 & 0.0000 & 0.0000 \\
\hline Hosking (50) & 188.043 & 187.970 & 205.352 & 203.574 \\
\hline p-Value & 0.7179321 & 0.7192047 & 0.3826575 & 0.4164732 \\
\hline Hosking $^{2}$ (50) & 207.557 & 202.431 & 192.019 & 197.642 \\
\hline p-Value & 0.3063950 & 0.3995570 & 0.6064352 & 0.4938025 \\
\hline Li-McLeod (50) & 188.272 & 188.175 & 205.438 & $203 ., 681$ \\
\hline p-Value & 0.7138843 & 0.7155944 & 0.3810407 & 0.4144023 \\
\hline Li-McLeod $^{2}(50)$ & 207.483 & 202.369 & 192.069 & 197.676 \\
\hline p-Value & 0.3076582 & 0.4007574 & 0.6054549 & 0.4931197 \\
\hline Akaike & 0.002791 & 0.002828 & 0.002845 & 0.002858 \\
\hline Schwarz & 0.024566 & 0.024603 & 0.024619 & 0.024633 \\
\hline
\end{tabular}

Figures 3 and 4 plot the conditional covariances for all the pairs of market returns during the whole period. We observe a tremble trend for all the conditional covariances. Additionally, conditional covariances seem to be extremely volatile. 
Figure 3 - Conditional Covariances of the Bivariate DCC-GARCH $(1,1)$ Model.

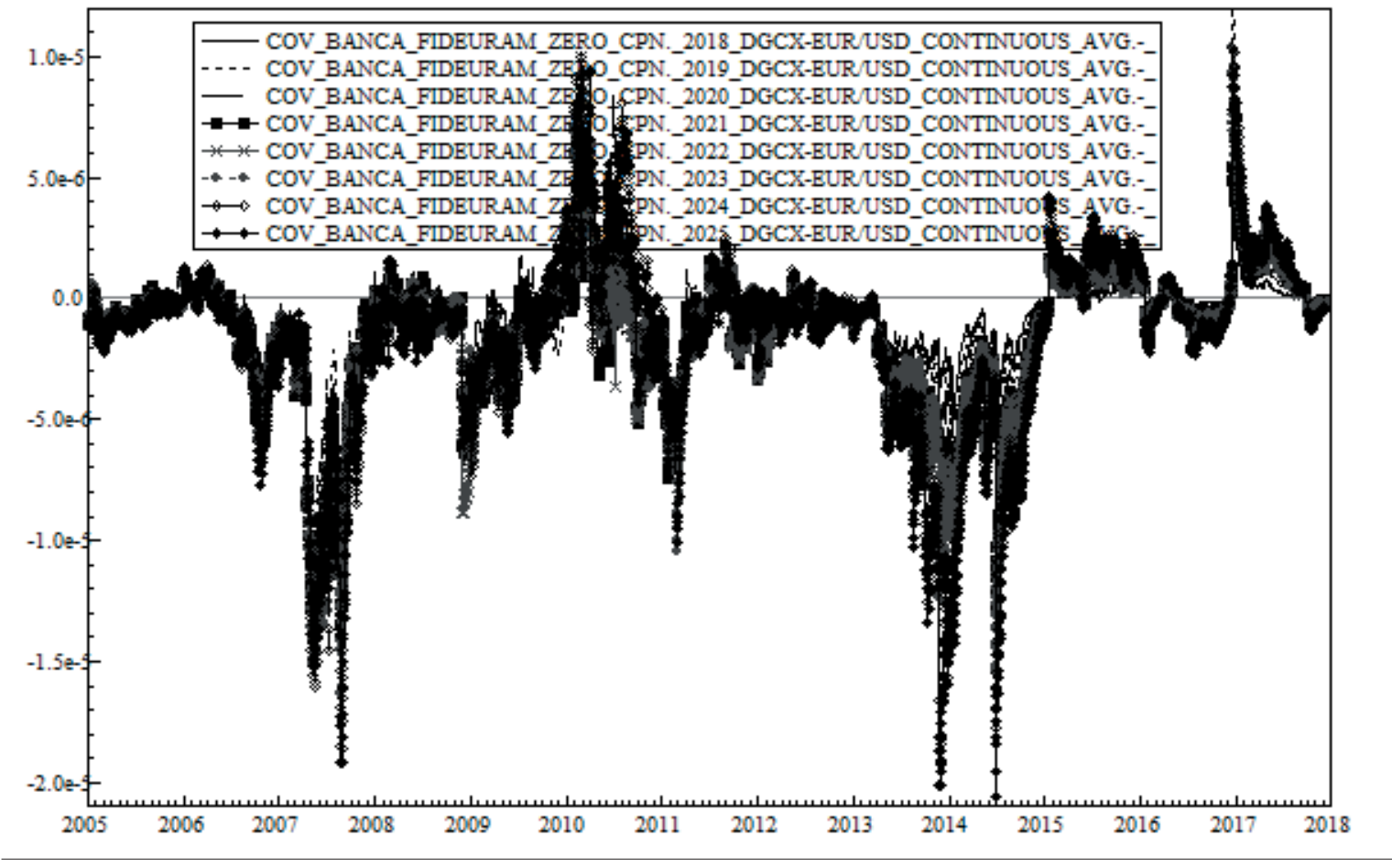

Figure 4 - Conditional Covariances of the Bivariate DCC-GARCH $(1,1)$ Model.

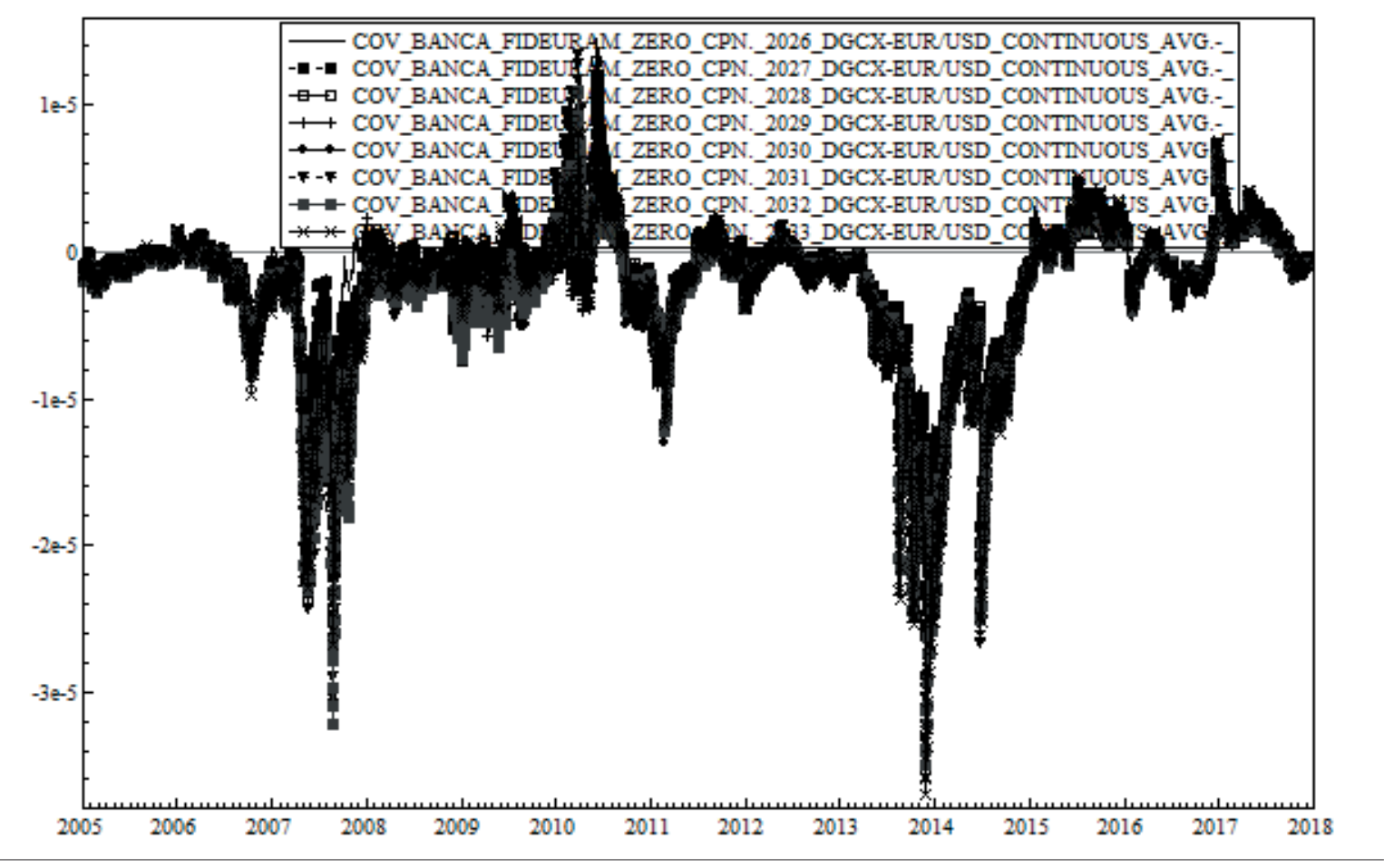




\section{ANALYSIS OF THE DYNAMIC CONDITIONAL CORRELATIONS (DCCS)}

Tables 17, 18, 19 and 20 show the descriptive statistics of the dynamic conditional correlations (DCCs) of the twenty-one pairs of markets generated by Equation 5 . We observe the highest mean value $(0,29828)$ for the pair of markets DGCX-EUR/USD CONTINUOUS AVG.- SETT. PRICE - BANCA FIDEURAM ZERO CPN. 2028. The highest std. deviation value for the pair of markets DGCX-EUR/USD CONTINUOUS AVG.- SETT. PRICE - BANCA FIDEURAM ZERO CPN. 2019 indicates that the specific DCC experiences larger fluctuations. The statistically significant Skewness, Excess Kyrtosis and the Jarque-Bera test statistics indicate that the DCCs for all the pairs of markets are not normally distributed.

Table 17 - Statistical Properties of the Multivariate GARCH-DCC's

\begin{tabular}{|c|c|c|c|c|}
\hline & $\begin{array}{c}\text { DGCX- } \\
\text { EUR/USD } \\
\text { CONTINUOUS } \\
\text { AVG.- SETT. } \\
\text { PRICE - BANCA } \\
\text { FIDEURAM } \\
\text { ZERO CPN. } \\
2018\end{array}$ & $\begin{array}{c}\text { DGCX- } \\
\text { EUR/USD } \\
\text { CONTINUOUS } \\
\text { AVG.- SETT. } \\
\text { PRICE - BANCA } \\
\text { FIDEURAM } \\
\text { ZERO CPN. } \\
2019\end{array}$ & $\begin{array}{c}\text { DGCX- } \\
\text { EUR/USD } \\
\text { CONTINUOUS } \\
\text { AVG.- SETT. } \\
\text { PRICE - BANCA } \\
\text { FIDEURAM } \\
\text { ZERO CPN. } \\
2020\end{array}$ & $\begin{array}{c}\text { DGCX- } \\
\text { EUR/USD } \\
\text { CONTINUOUS } \\
\text { AVG.- SETT. } \\
\text { PRICE - BANCA } \\
\text { FIDEURAM } \\
\text { ZERO CPN. } \\
2021\end{array}$ \\
\hline Mean & -0.039373 & -0.042172 & -0.03832 & -0.043446 \\
\hline Minimum & -0.35803 & -0.36773 & -0.33772 & -0.33122 \\
\hline Maximum & 0.16949 & 0.19866 & 0.15557 & 0.13102 \\
\hline Std. Deviation & 0.086969 & 0.088029 & 0.083352 & 0.081366 \\
\hline Skewness & $-0.66274^{\star * *}$ & $-0.59605^{\star * *}$ & $-0.77505^{\star \star *}$ & $-0.86371^{\star * *}$ \\
\hline p-Value & $1.0141 \mathrm{e}-055$ & $2.0647 \mathrm{e}-045$ & $1.5787 \mathrm{e}-075$ & $2.4504 \mathrm{e}-093$ \\
\hline Excess Kyrtosis & $0.77644^{* * *}$ & $0.67404^{* * *}$ & $0.74112^{* * *}$ & $0.71865^{\star * *}$ \\
\hline p-Value & $3.1330 \mathrm{e}-020$ & $1.2544 \mathrm{e}-015$ & $1.4292 \mathrm{e}-018$ & $1.4835 \mathrm{e}-017$ \\
\hline Jarque-Bera & $331.84^{* * *}$ & $263.73^{* * *}$ & $415.13^{* * *}$ & $492.25^{* * *}$ \\
\hline p-Value & $8.7272 \mathrm{e}-073$ & $5.3801 \mathrm{e}-058$ & 7.1627e-091 & $1.2891 \mathrm{e}-107$ \\
\hline Akaike & 0.002791 & 0.002828 & 0.002845 & 0.002858 \\
\hline Schwarz & 0.024566 & 0.024603 & 0.024619 & 0.024633 \\
\hline
\end{tabular}


Table 18 - Statistical Properties of the Multivariate GARCH-DCC's

\begin{tabular}{|c|c|c|c|c|}
\hline & $\begin{array}{c}\text { DGCX- } \\
\text { EUR/USD } \\
\text { CONTINUOUS } \\
\text { AVG.- SETT. } \\
\text { PRICE - BANCA } \\
\text { FIDEURAM } \\
\text { ZERO CPN. } \\
2022\end{array}$ & $\begin{array}{c}\text { DGCX- } \\
\text { EUR/USD } \\
\text { CONTINUOUS } \\
\text { AVG.- SETT. } \\
\text { PRICE - BANCA } \\
\text { FIDEURAM } \\
\text { ZERO CPN. } \\
2023\end{array}$ & $\begin{array}{c}\text { DGCX- } \\
\text { EUR/USD } \\
\text { CONTINUOUS } \\
\text { AVG.- SETT. } \\
\text { PRICE - BANCA } \\
\text { FIDEURAM } \\
\text { ZERO CPN. } \\
2024\end{array}$ & $\begin{array}{c}\text { DGCX- } \\
\text { EUR/USD } \\
\text { CONTINUOUS } \\
\text { AVG.- SETT. } \\
\text { PRICE - BANCA } \\
\text { FIDEURAM } \\
\text { ZERO CPN. } \\
2025\end{array}$ \\
\hline Mean & -0.044479 & -0.036367 & -0.03233 & -0.043351 \\
\hline Minimum & -0.29975 & -0.32593 & -0.32002 & -0.31119 \\
\hline Maximum & 0.13255 & 0.15368 & 0.16649 & 0.1375 \\
\hline Std. Deviation & 0.075008 & 0.078369 & 0.086434 & 0.079562 \\
\hline Skewness & $-0.59792^{\star * \star}$ & $-0.61477^{\star * *}$ & $-0.61657^{\star * *}$ & $-0.70375^{\star * *}$ \\
\hline p-Value & $1.0982 \mathrm{e}-045$ & $3.3949 \mathrm{e}-048$ & $1.8166 \mathrm{e}-048$ & $1.3480 \mathrm{e}-062$ \\
\hline Excess Kyrtosis & $0.46461^{\star \star \star}$ & $0.67357^{\star \star *}$ & $0.40503^{\star * \star}$ & $0.70271^{\star \star *}$ \\
\hline p-Value & $3.5133 \mathrm{e}-008$ & $1.3114 \mathrm{e}-015$ & $1.5354 \mathrm{e}-006$ & $7.4775 e-017$ \\
\hline Jarque-Bera & $231.46^{* * *}$ & $276.40^{* * *}$ & $236.91^{\star * *}$ & $348.03^{\star * *}$ \\
\hline p-Value & $5.4935 \mathrm{e}-051$ & $9.5830 \mathrm{e}-061$ & $3.6013 \mathrm{e}-052$ & $2.6732 \mathrm{e}-076$ \\
\hline
\end{tabular}

Table 19 - Statistical Properties of the Multivariate GARCH-DCC's

\begin{tabular}{|c|c|c|c|c|}
\hline & $\begin{array}{c}\text { DGCX- } \\
\text { EUR/USD } \\
\text { CONTINUOUS } \\
\text { AVG.- SETT. } \\
\text { PRICE - BANCA } \\
\text { FIDEURAM } \\
\text { ZERO CPN. } \\
2026\end{array}$ & $\begin{array}{c}\text { DGCX- } \\
\text { EUR/USD } \\
\text { CONTINUOUS } \\
\text { AVG.- SETT. } \\
\text { PRICE - BANCA } \\
\text { FIDEURAM } \\
\text { ZERO CPN. } \\
2027\end{array}$ & $\begin{array}{c}\text { DGCX- } \\
\text { EUR/USD } \\
\text { CONTINUOUS } \\
\text { AVG.- SETT. } \\
\text { PRICE - BANCA } \\
\text { FIDEURAM } \\
\text { ZERO CPN. } \\
2028\end{array}$ & $\begin{array}{c}\text { DGCX- } \\
\text { EUR/USD } \\
\text { CONTINUOUS } \\
\text { AVG.- SETT. } \\
\text { PRICE - BANCA } \\
\text { FIDEURAM } \\
\text { ZERO CPN. } \\
2029\end{array}$ \\
\hline Mean & -0.033146 & -0.042371 & -0.032106 & -0.042044 \\
\hline Minimum & -0.29929 & -0.28349 & -0.25206 & -0.2736 \\
\hline Maximum & 0.11153 & 0.092663 & 0.10489 & 0.096035 \\
\hline Std. Deviation & 0.077377 & 0.071889 & 0.0673 & 0.069413 \\
\hline Skewness & $-0.96329^{\star * *}$ & $-0.95533^{* * *}$ & $-0.87138^{\star \star *}$ & $-0.93736^{\star * *}$ \\
\hline p-Value & $1.2552 \mathrm{e}-115$ & $9.3446 \mathrm{e}-114$ & $5.7226 \mathrm{e}-095$ & $1.3692 \mathrm{e}-109$ \\
\hline Excess Kyrtosis & $0.93473^{\star * *}$ & $0.91733^{* * *}$ & $0.87606^{* * *}$ & $1.0724^{\star * \star}$ \\
\hline p-Value & $1.3611 \mathrm{e}-028$ & $1.3410 \mathrm{e}-027$ & $2.5739 \mathrm{e}-025$ & $4.2329 \mathrm{e}-037$ \\
\hline Jarque-Bera & $644.83^{* \star *}$ & $631.70^{\star \star \star}$ & $535.03^{\star * \star}$ & $655.95^{\star * *}$ \\
\hline p-Value & $9.4906 \mathrm{e}-141$ & $6.7291 \mathrm{e}-138$ & $6.5873 e-117$ & $3.6472 \mathrm{e}-143$ \\
\hline
\end{tabular}


Table 20 - Statistical Properties of the Multivariate GARCH-DCC's

\begin{tabular}{|c|c|c|c|c|}
\hline & $\begin{array}{c}\text { DGCX- } \\
\text { EUR/USD } \\
\text { CONTINUOUS } \\
\text { AVG.- SETT. } \\
\text { PRICE - BANCA } \\
\text { FIDEURAM } \\
\text { ZERO CPN. } \\
2030\end{array}$ & $\begin{array}{c}\text { DGCX- } \\
\text { EUR/USD } \\
\text { CONTINUOUS } \\
\text { AVG.- SETT. } \\
\text { PRICE - BANCA } \\
\text { FIDEURAM } \\
\text { ZERO CPN. } \\
2031\end{array}$ & $\begin{array}{c}\text { DGCX- } \\
\text { EUR/USD } \\
\text { CONTINUOUS } \\
\text { AVG.- SETT. } \\
\text { PRICE - BANCA } \\
\text { FIDEURAM } \\
\text { ZERO CPN. } \\
2032\end{array}$ & $\begin{array}{c}\text { DGCX- } \\
\text { EUR/USD } \\
\text { CONTINUOUS } \\
\text { AVG.- SETT. } \\
\text { PRICE - BANCA } \\
\text { FIDEURAM } \\
\text { ZERO CPN. } \\
2033\end{array}$ \\
\hline Mean & -0.038035 & -0.037904 & -0.043152 & -0.037287 \\
\hline Minimum & -0.25894 & -0.26307 & -0.25104 & -0.25792 \\
\hline Maximum & 0.095731 & 0.10924 & 0.097284 & 0.10538 \\
\hline Std. Deviation & 0.065429 & 0.06739 & 0.064469 & 0.068924 \\
\hline Skewness & $-0.87481^{\star * *}$ & $-0.79454^{\star * *}$ & $-0.86477^{\star * *}$ & $-0.92264^{\star * *}$ \\
\hline p-Value & $1.0575 \mathrm{e}-095$ & $2.7998 \mathrm{e}-079$ & $1.4595 \mathrm{e}-093$ & $3.0921 \mathrm{e}-106$ \\
\hline Excess Kyrtosis & $0.87804^{* * *}$ & $0.90575^{\star * *}$ & $1.0857^{\star * *}$ & $1.0885^{* * *}$ \\
\hline p-Value & $2.0112 \mathrm{e}-025$ & $6.0021 \mathrm{e}-027$ & $5.4860 \mathrm{e}-038$ & $3.5911 \mathrm{e}-038$ \\
\hline Jarque-Bera & $538.89^{* * *}$ & $470.47^{\star * *}$ & $586.42^{\star * *}$ & $645.45^{* * *}$ \\
\hline p-Value & $9.5881 \mathrm{e}-118$ & $6.8992 \mathrm{e}-103$ & $4.5696 \mathrm{e}-128$ & $6.9584 \mathrm{e}-141$ \\
\hline
\end{tabular}

Figures 5 and 6 present the pair-wise Dynamic Conditional Correlations (DCCs). We observe strong co-movements for all DCCs. DCCs have positive values in sub-periods, indicating the existence of contagion, implying the specific correlations risky for any investor. Furthermore, we can notice the effects of major economic events on the DCC graphs as we see that the lines are bouncing above and beyond, i.e. (a) the bankruptcy of Lehman Brothers (14/09/2008), (b) the European Central Bank announcement of an aggressive money-creation program, printing more than one trillion new euros (22/01/2015), (c) Black Monday (24/08/2015), and (d) the United Kingdom referendum (23/06/2016), among others.

Figure 5 - Dynamic Conditional Correlations for All the Pairs of Markets Generated by the Bivariate DCC-GARCH(1,1) Model.

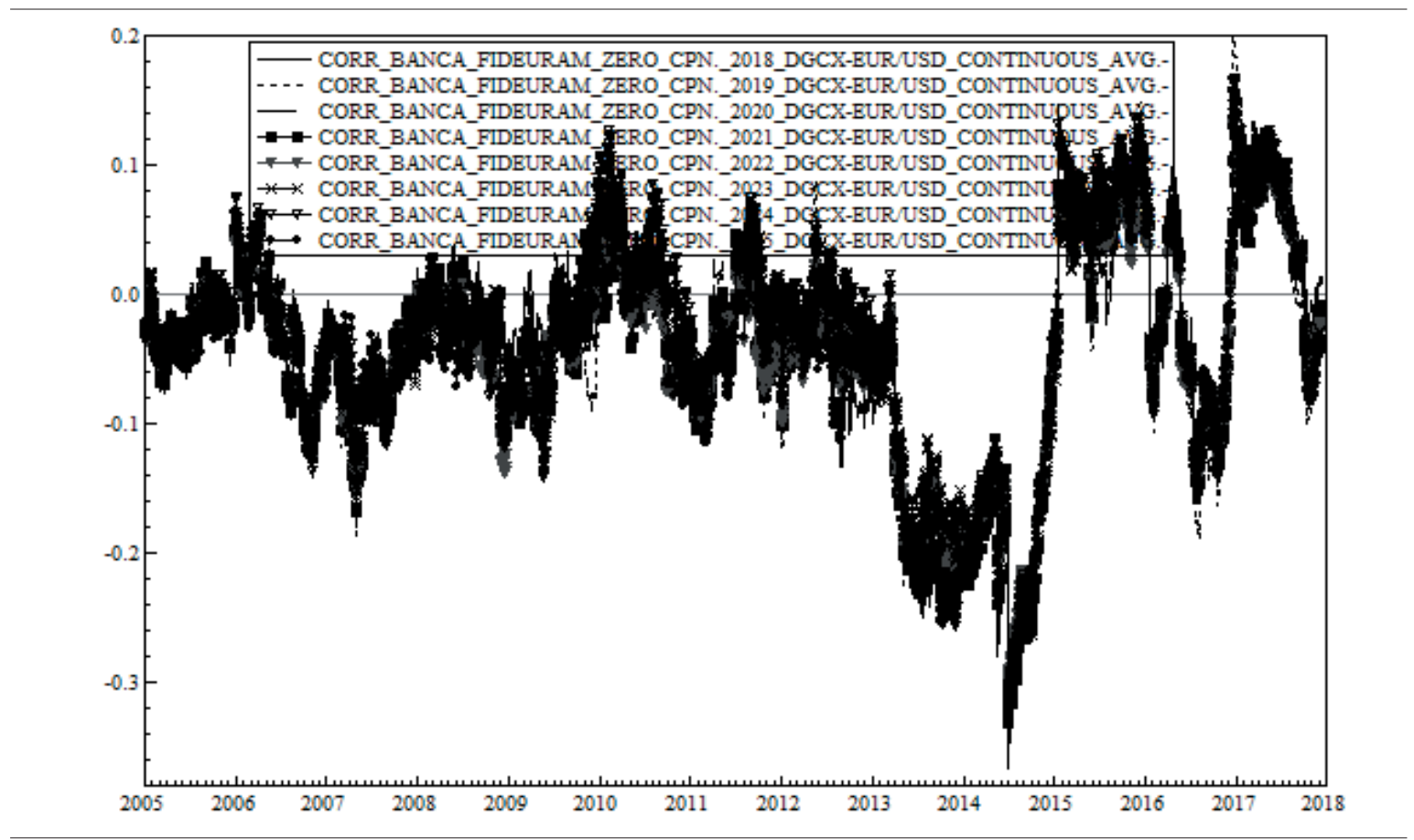


Figure 6 - Dynamic Conditional Correlations for All the Pairs of Markets Generated by the Bivariate DCC-GARCH(1,1) Model.

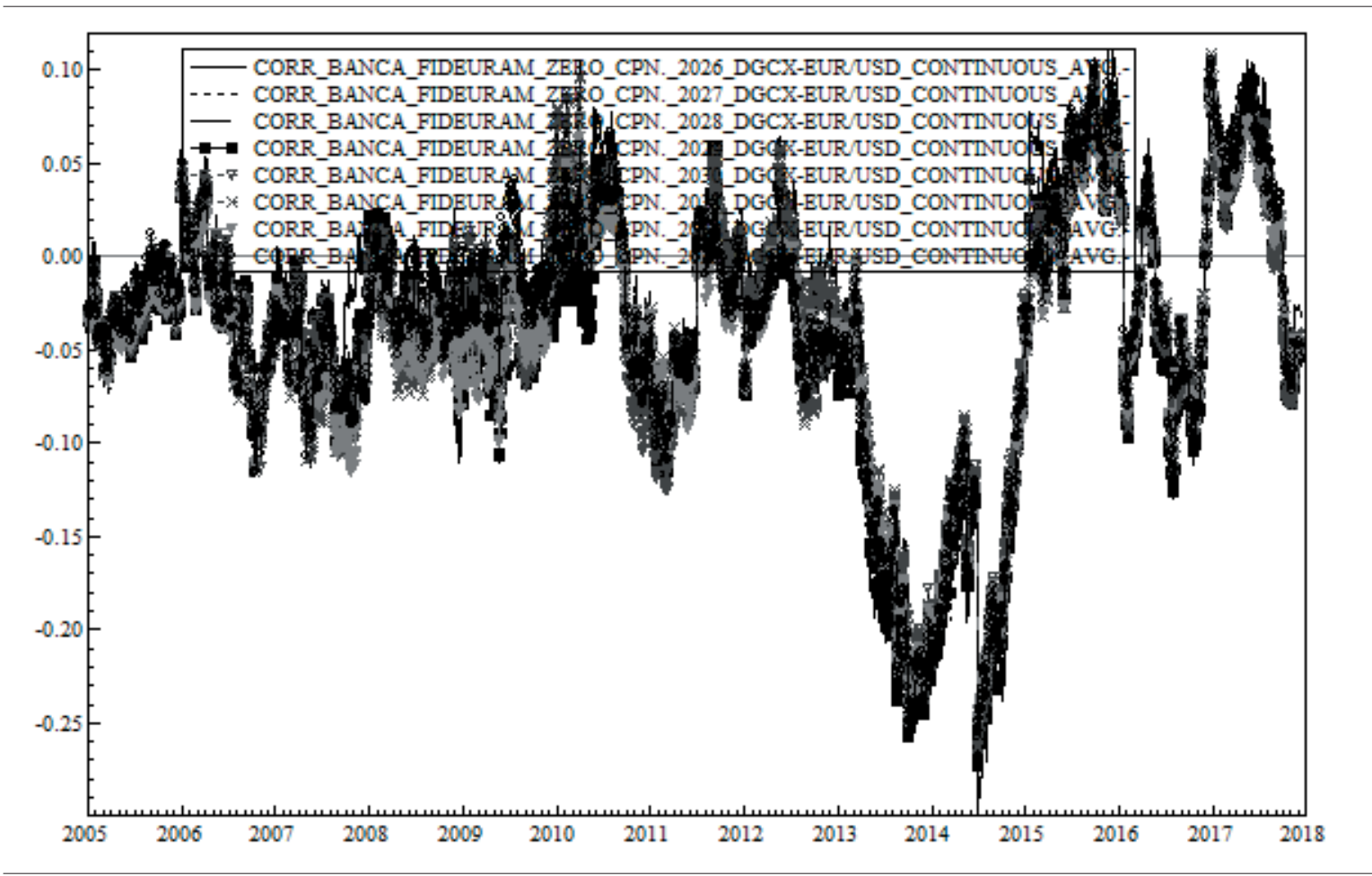

\section{CONCLUSIONS}

This paper investigates the potential volatility spillovers effects and the existence of contagion effects of the Eurodollar futures market and sixteen zero coupons of Banca Fideuram by employing a bivariate DCC-GARCH model. We set the under-investigation period from 2005 until 2017. To the best of our knowledge, this is the first empirical study investigating volatility spillovers between the Eurodollar futures market and the zero coupons of Banca Fideuram.

The main empirical results are summarized as follows. Based on the descriptive statistics, BANCA FIDEURAM ZERO CPN. 2032 returns present the largest fluctuations compared to the rest markets. Furthermore, results of the bivariate DCC-GARCH model indicate strong evidence of volatility spillover effects. DCCs analysis shows evidence of strong co-movements for all the pairs of markets. Additionally, DCCs reveal contagion for all the pairs of markets in sub-periods. The empirical results are of interest to policymakers, who provide regulations for the under-investigation derivative markets, as well as to market-makers.

\section{ACKNOWLEDGMENTS}

This article was carried out by me independently. The research is original, and has not been submitted to any other journal. I want to thank the anonymous referees for their valuable comments and suggestions which helped me to improve the paper. Any responsibility for remaining errors in the resulting work is my own. 


\section{REFERENCES}

Aboura, S., \& Chevallier, J. (2015). Volatility returns with vengeance: financial markets vs. commodities. Research in International Business and Finance 33, 334-354. DOI: 10.1016/j.ribaf.2014.04.003.

Antonakakis, N., \& Kizys, R. (2015). Dynamic spillovers between commodity and currency markets. International Review of Financial Analysis 41, 303-319. DOI: 10.1016/j.irfa.2015.01.016.

Antonakakis, N., Floros, C., \& Kizys, R. (2016). Dynamic spillover effects in futures markets: UK and US evidence. International Review of Financial Analysis 48, 406-418. DOI: 10.1016/j.irfa.2015.03.008.

Bagchi, B. (2017). Volatility spillovers between crude oil price and stock markets: evidence from BRIC countries. International Journal of Emerging Markets, 12(2), 352-365. DOI: 10.1108/IJoEM-04-2015-0077.

Belhassine, O. (2020). Volatility spillovers and hedging effectiveness between the oil market and Eurozone sectors: A tale of two crises. Research in International Business and Finance 53. DOI: 10.1016/j.ribaf.2020.101195.

Bollerslev, T. (1986). Generalized autoregressive conditional heteroskedasticity. Journal of Econometrics 31(3), 307-327. DOI: 10.1016/0304-4076(86)90063-1.

Chang, C.L., McAleer, M., \& Wang, Y. (2018). Testing Co-Volatility spillovers for natural gas spot, futures and ETF spot using dynamic conditional covariances. Energy 151, 984-997. DOI: 10.1016/j.energy.2018.01.017.

Dickey, D. A., \& Fuller, W. A. (1979). Distribution of the Estimators for Autoregressive Time Series with a Unit Root. Journal of the American Statistical Association 74, 427-431. DOI: 10.2307/2286348.

Dimitriou, D. and Kenourgios, D. (2015). Contagion of the global financial crisis and the real economy: A regional analysis. Economic Modelling 44, 283-293. DOI: 10.1016/j.econmod.2014.10.048.

Dimitriou, D, Kenourgios, D., \& Simos, T. (2013). Global financial crisis and emerging stock market contagion: A multivariate FIAPARCH-DCC approach. International Review of Financial Analysis 30, 46-56. DOI: 10.1016/j.irfa.2013.05.008.

Du, L., \& He, Y. (2015). Extreme risk spillovers between crude oil and stock markets. Energy Economics 51, 455-465. DOI: 10.1016/j.eneco.2015.08.007.

Efimova, O., \& Serletis, A. (2014). Energy markets volatility modelling using GARCH. Energy Economics 43, 264-273. DOI: 10.1016/j.eneco.2014.02.018.

Engle, R. F. (2002). Dynamic conditional correlation-a simple class of multivariate GARCH models. Journal of Business \& Economic Statistics 20, 339-350. DOI: 10.1198/073500102288618487.

Ewing, B.T., \& Malik, F. (2016). Volatility spillovers between oil prices and the stock market under structural breaks. Global Finance Journal 29, 12-23. DOI: 10.1016/j.gf.2015.04.008.

Forbes, K., \& Rigobon, R. (2002). No contagion, Only Interdependence: Measuring Stock Market CoMovements. Journal of Finance, 57, 2223-2261. DOI: 10.1111/0022-1082.00494.

Hosking, J. R. M. (1980). The Multivariate Portmanteau Statistic. Journal of the American Statistical Association 75(371), 602-608. DOI: 10.2307/2287656.

Hu, H., Chen, D., Sui, B., Zhang, L., \& Wang, Y. (2020). Price volatility spillovers between supply chain and innovation of financial pledges in China. Economic Modelling 89, 397-413. DOI: 10.1016/j.econmod.2019.11.012.

Kavussanos, M.G., Visvikis, I.D., \& Dimitrakopoulos, D.N. (2014). Economic spillovers between related derivatives markets: The case of commodity and freight markets. Transportation Research Part E: Logistics and Transportation Review 68, 79-102. DOI: 10.1016/j.tre.2014.05.003.

Li, K.X., Qi, G., Shi, W., Yang, Z., Bang, H.S., Woo, S.H., \& Yip, T.L. (2014). Spillover effects and dynamic correlations between spot and forward tanker freight markets. Maritime Policy \& Management 41(7), 683-696. DOI: $10.1080 / 03088839.2014 .958585$.

Li, Y., \& Giles, D. E. (2015). Modelling volatility spillover effects between developed stock markets and Asian emerging stock markets. International Journal of Finance and Economics 20, 155-177. DOI: 10.1002/ijfe.1506.

McLeod, A. I., \& Li, W. K. (1983). Diagnostic checking ARMA time series models using squared-residuals autocorrelations. Journal of Time Series Analysis 4(4), 269-273. DOI: 10.1111/j.1467-9892.1983.tb00373.x. 
Ma, Y.R., Zhang, D., Ji, Q., \& Pan, J. (2019). Spillovers between oil and stock returns in the US energy sector: Does idiosyncratic information matter? Energy Economics 81, 536-544. DOI: 10.1016/j.eneco.2019.05.003.

Mensi, W., Beljid, M., Boubaker, A., \& Managi, S. (2013). Correlations and volatility spillovers across commodity and stock markets: Linking energies, food, and gold. Economic Modelling 32, 15-22. DOI: 10.1016/j.econmod.2013.01.023.

Roy, R.P., \& Roy, S.S. (2017). Financial contagion and volatility spillover: An exploration into Indian commodity derivative market. Economic Modelling 67, 368-380. DOI: 10.1016/j.econmod.2017.02.019.

Sehgal, S., Ahmad W., \& Deisting, F. (2015). An investigation of price discovery and volatility spillovers in India's foreign exchange market. Journal of Economic Studies 42(2), 261-284. ISSN: 0144-3585.

Sensoy, A., Hacihasanoglu, E., \& Nguyen, D.K., (2015). Dynamic convergence of commodity futures: Not all types of commodities are alike. Resources Policy 44(3). DOI: 10.1016/j.resourpol.2015.03.001.

Singhal S., \& Ghosh, S. (2016). Returns and volatility linkages between international crude oil price, metal and other stock indices in India: Evidence from VAR-DCC-GARCH models. Resources Policy. Vol. 50(3), 276-288 (2016) DOI: 10.1016/j.resourpol.2016.10.001.

Sukhonpitumart, P., Jaroenjitrkam, A., Maneenop, S., \& Padungsaksawasdi, C. (2020). RETURN AND VOLATILITY SPILLOVERS BETWEEN STOCK AND FUTURES MARKETS IN THAILAND. Academy of Accounting and Financial Studies Journal 24(2), 1-14.

Sun, X., Haralambides, H., \& Liu, H. (2019). Dynamic spillover effects among derivative markets in tanker shipping. Transportation Research Part E: Logistics and Transportation Review 122, 384-389. DOI: $10.1016 /$ j.tre.2018.12.018.

Tsiaras, K., \& Simos, T. (2020). FOREX and equity markets spillover effects among USA, Brazil, Italy, Germany and Canada in the aftermath of the Global Financial Crisis. Journal of Finance and Accounting Research 2(1). DOI: $10.32350 /$ JFAR/0201/03.

Tsiaras, K. (2020). Dynamic relationship between future FOREX markets in the post Global Financial Crisis. Journal of Quantitative Methods 4(1), 30-52. DOI: 10.29145/2020/jqm/040102.

Tsiaras, K. (2020). Contagion in crude oil future market and 3Y, 4Y and 5Y CDS markets for the post-Global Financial Crisis: A multivariate GARCH-cDCC approach. Ekonomická revue. Accepted Paper for publication in the upcoming issue.

Yu, L., Zha, R., Stafylas, D., He, K., \& Liu, J. (2020). Dependences and volatility spillovers between the oil and stock markets: New evidence from the copula and VAR-BEKK-GARCH models. International Review of Financial Analysis 68. DOI: 10.1016/j.irfa.2018.11.007. 


\section{Rezime:}

Ovaj rad ispituje vremenski različite uslovne korelacije između terminskog tržišta Eurodollar i nula kupona Banca Fideuram. Primenjujemo GARCH model bivarijantne dinamičke uslovne korelacije (DCC) kako bismo zabeležili potencijalne efekte zaraze između tržišta za period 2005-2017. Empirijski rezultati otkrivaju zarazu tokom istražnog perioda u vezi sa dvadeset i jednim bivarijantnim modelom, pokazujući da tržište futura Eurodollar ima veliki uticaj na nulte kupone Banca Fideuram. Nalazi imaju presudne implikacije za kreatore politika koji pružaju propise za gore navedena tržišta derivata.
Ključne reči:

DCC-GARCH model, buduće tržište EURODOLLAR, nula kupona, finansijska zaraza, dinamičke uslovne korelacije.

Klasifikacija jela:

C58, C61, G11, G15. 